\title{
Dynamics of the Ion-Ion Acoustic Instability in the Thermalization of Ion Beams
}

\author{
J.H. Han, W. Horton, and J.N. Leboeuf ${ }^{a}$ \\ Institute for Fusion Studies \\ The University of Texas at Austin \\ Austin, Texas 78712
}

DOE/ET/53088--562

DE92 019396

\begin{abstract}
Particle simulation using a nonlinear adiabatic electron response with two streaming ion species and nonlinear theory are used to study the collisionless thermalization of ion beams in a hot electron plasma. The slow beam or subsonic regime is investigated and the criterion for the transition from predominantly light ion to predominantly heavy ion heating is developed. Long-lived ion hole structures are observed in the final state.
\end{abstract}

\section{DISCLAIMER}

\begin{abstract}
This report was prepared as an account of work sponsored by an agency of the United States Government. Neither the United States Government nor any agency thercof, nor any of their employees, makes any warranty, express or implied, or assumes any legal liability or responsibility for the accuracy, completeness, or usefulness of any information, apparatus, product, or process disclosed, or represents that its use would not infringe privately owned rights. Refer. ence herein to any specific commervial product, prosess, or service by trade name, trademark, manufacturer, or otherwise does not necessarily constitute or imply its endorsement, recom. mendation, or favoring by the United States Government or any agency thereof. The views and opinions of authors expressed herein do not necessarily state or reflect those of the
United States Government or any agency thercof.
\end{abstract}

a) Oak Ridge National Laboratory, P.O. Box 2009, Oak Ridge, TN 37831 


\section{Introduction}

In a plasma with a potential sheath or double layers, such as occurring along auroral magnetic field lines in the upper ionosphere, ion species with different charge to mass ratios are accelerated to different strearning velocities. The streaming velocities of the ions can easily approach the ion acoustic speed since the sheath and double layer potential drops are determined by the electron temperature. When the plasma source of the accelerated ion is at a low temperature, as in the case of the ionosphere, this acceleration mechanism naturally leads to the generation of ion beams with velocity spreads small compared with their streaming velocities. Such multiple cold ion streams are unstable to the ion-ion acoustic instability. Satellites observations ${ }^{1,2}$ shows that $\mathrm{O}^{+}$ions are preferentially heated to $\mathrm{H}^{+}$ions within the auroral cavity region. This linear ion-ion acoustic instability or ion-streaming instability leads to wave generation and to ion-heating problems within the magnetosphere, and there is a growing field of interest with numerous recent warks are being published.

Bergmann and Lotto ${ }^{3}$ studied the statility of $\mathrm{O}^{+}-\mathrm{H}^{+}$outflows in the ionosphere and reported that the $\mathrm{O}^{+}-\mathrm{H}^{+}$two stream instability is a key mechanjsm for the heating of the upflowing auroral ions. Dusenberry and Martin ${ }^{4}$ did a numerical study using various particle distribution models for the auroral ions and characterized the excited mode either nonresonant ion-jon two stream mode or resonant acoustic mode and suggest that wave-particle interactions with these waves are responsible for the parallel temperature heating of the auroral ions. Subsequently, Dusenberwy, Martin, and Winglee extended their previous work to oblique modes and carried out linear analysis varying the relative ion concentration, ion mass, and $T_{e} / T_{i}$. Dum ${ }^{6}$ carried out extensive linear and quasi-linear analyses of beam-plasma and two stream instability from the hydrodynamic to the kinetic regime which is applicable to instabilities with ion beams. Winglee et al. ${ }^{7}$ studied the heating of ion beams produced 
by ion-ion acoustic instability by using 2-D particle code with artificial electron-ion mass ratio $m_{e} / m_{i}$ and explained the heating as the net transfer of energy from the light ions to the heavy ions.

More recently Schriver et al. ${ }^{8}$ and Schriver and Ashour-Abdalla ${ }^{9}$ both performed particle simulation using guiding-center particle code with purpose of finding the range of the nonlinear behavior of the ion-ion acoustic modes for varying the most relevant physical parameters in the auroral zone $e^{8}$ and in the plasma sheet boundary layer respectively. ${ }^{9}$ Chen and Ashour-Abdalla ${ }^{10}$ also applied the same approaches to study the heating of the polar wind. Karimabadi, Omidi and Quest ${ }^{11}$ carried out a simulation using 2-D hybrid-particle code and reported the saturation mechanism of the ion-ion acoustic instability is ion trapping in the two degenerate obliquely propagating waves.

Here we study numerically and analytically the nonlinear evolution of the ion-ion acoustic turbulence along with the linear analysis. We considered the weakly unstable or subsonic case where only the parallel propagating one dimensional turbulent heating is prominent. We consider the diversion of the free energy from the streaming ions into the heavy and light ion species. We attempt to clarify the three stages of the evolution and give several formulas for the types of collective thermalizations occurring in subsonic regime where the electrons have a substantially high temperature. We obtained the results using a semi-implicit hybrid particle code that allows the use of massless electrons. The semi-implicit time stepping allows the study of the long-time behaviour where we report the coalescence instability results in the formation of a few large scale structures. The final long time structures appear to be the phase space holes of Dupree. ${ }^{12}$ 


\section{Ion-Ion Acoustic Wave Stability and Simulation Equations}

\section{A. Linear Stability Analyses}

The linear kinetic dispersion relation follows from the linearized Vlasov equations for the two ion species and the electrons. One ion species is designated as light $(L)$ and the second species as heavy $(\mathrm{H})$ where in the numerical examples we consider the case of hydrogen $\left(\mathrm{H}^{+}\right)$and the heavy ion species of singly ionized oxygen $\left(\mathrm{O}^{+}\right)$. By following the standard procedures the linear kinetic dispersion relation governing the waves and their stability is given by

$$
\varepsilon(k, \omega)=1+\frac{1}{k^{2} \lambda_{D e}^{2}}\left[1+\xi_{e} Z\left(\xi_{e}\right)\right]+\frac{1}{k^{2} \lambda_{D L}^{2}}\left[1+\xi_{i L} Z\left(\xi_{i L}\right)\right]+\frac{1}{k^{2} \lambda_{D H}^{2}}\left[1+\xi_{i H} Z\left(\xi_{i H}\right)\right]=0
$$

where the argument of plasma dispersion function $Z(\xi)$ for electron, light ions and heavy ions are

$$
\xi_{e}=\frac{\omega}{\sqrt{2} k v_{T e}}, \xi_{i L}=\frac{\omega-k v_{D L}}{\sqrt{2} k v_{T i L}}, \xi_{i H}=\frac{\omega-k v_{D H}}{\sqrt{2} k v_{T i H}}
$$

and the associated Debye shielding lengths are

$$
\lambda_{D e}^{2}=\frac{T_{e}}{4 \pi n_{0} e^{2}}, \lambda_{D L}^{2}=\frac{T_{i}}{4 \pi n_{O L} e^{2}}, \lambda_{D H}^{2}=\frac{T_{i}}{4 \pi n_{O H} e^{2}} .
$$

Here $v_{D L}, v_{D H}$ are the light and heavy ion strearning velocities with respect to the laboratory rest frame in which the electron drift velocity, if any, is a negligible effect. For brevity, we will also introduced the subscript 1 for the light ion quantities and 2 for the heavy ion quantities.

For the ion-ion acoustic instability it is appropriate to introduce the space time normalization $\tilde{\omega}=\omega / \omega_{p L}$ and $\tilde{k}=k \lambda_{D e}$, where $\omega_{p L}$ is the light ion plasma frequency. The normalized kinetic dispersion relation can be written as

$$
\begin{aligned}
\varepsilon(\tilde{k}, \tilde{\omega}) & =1+\frac{1}{\tilde{k}^{2}}\left[1+\tilde{\xi}_{e} Z\left(\tilde{\xi}_{e}\right)\right]+\frac{n_{i L}}{n_{0}} \frac{\tau}{\tilde{k}^{2}}\left[1+\tilde{\xi}_{i L} Z\left(\tilde{\xi}_{i L}\right)\right] \\
& +\frac{n_{i H}}{n_{0}} \frac{m_{i L}}{m_{i H}} \frac{\tau}{\tilde{k}^{2}}\left[1+\tilde{\xi}_{i H} Z\left(\tilde{\xi}_{i H}\right)\right]
\end{aligned}
$$


where $n_{i L}, n_{i H}$ is the equilibrium number density of light and heavy ion species and $\tau=T_{e} / T_{i}$. This kinetic dispersion relation is compared with the hot electron-cold ion fluid dispersion relation in the limit of

$$
\tilde{\xi}_{i L}, \tilde{\xi}_{i H} \gg 1 \quad \text { and } \quad \tilde{\xi}_{e} \ll 1
$$

where the dispersion relation (4) becomes

$$
\varepsilon(\tilde{k}, \tilde{\omega})=1+\frac{1}{\tilde{k}^{2}}-\frac{1}{\left(\tilde{\omega}-\tilde{k} \tilde{v}_{D L}\right)^{2}}-\frac{m_{L}}{m_{H}} \frac{n_{i H}}{n_{i L}} \frac{1}{\left(\tilde{\omega}-\tilde{k} \tilde{v}_{D H}\right)^{2}}=0,
$$

where velocities $\tilde{v}_{D L}, \tilde{v}_{D H}$ are normalized by $\lambda_{D e} \omega_{p L}$. We define the hydrodynamic beam stability parameter $\eta$ as

$$
\eta=\frac{n_{i H}}{n_{i L}} \frac{m_{L}}{m_{H}} \equiv \frac{n_{2} m_{1}}{n_{1} m_{2}} .
$$

which is less than unity due to $m_{2} \gg m_{1}$ unless there is only a trace of the light ions.

The principal rocts of the kinetic dispersion Eq. (4) are shown in Fig. 1 for the reference parameters given in Table I. Four separate branches of kinetic branches are found. Among these four principal roots there exist only one unstable mode (denoted as I) and there is also one marginally stable mode (denoted as III) for wavenumbers from $k \lambda_{D e}=0.196$ to 2.50 . The other two roots (II, IV) in Fig. 1 are damped for all wavenumbers. For comparison, we solve the dispersion relations in the fluid limit where there are also four fluid modes which can be matched with corresponding kinetic modes in Fig. 2. The analyses shows that in the fluid limit, modes I, II, and IV have finite imaginary part while mode III has zero imaginary part for a wavenumber from $k \lambda_{D e}=0.431$ to 2.384 . The three modes (I, II, and IV) with finite imaginary part have degenerate real frequencies for a wavenumber which give zero imaginary part for mode III. The unstable kinetic and fluid modes are compared in Fig. 3. The kinetic mode has a smaller growth rate than the fluid mode due to the linear ion Landau damping but a wider range of unstable wavelengths than the fluid mode.

For large $k \lambda_{D e}$ the fluid mode I has a real frequency above $\omega_{p L}$, where $\omega_{p L}$ is the light ion plasma frequency. To see how the growth rate of the most unstable mode varies with 
the light ion fractional population density the linear dispersion relation (4) is solved for the $n_{0 L} / n_{0}$ parameter variation. Fig. 4 shows that growth rate has its maximum when light ion. species has equal density with heavy ion species. In Fig. 4 the mass ratio between heavy and light ion species is kept as $m_{H} / m_{L}=16$. Another parameter variation of interest is the mass ratio $\left(m_{L} / m_{H}\right)$. The linear analysis results for the heavy ion mass ratio variation while light ion mass is fixed are given in Figs. 5 and 6 showing that the growth rate has a logarithmic dependence on $m_{e} / m_{H}$.

\section{B. Simulation model and the Algorithm}

The particle simulation code used to study the evolution of the two-ion acoustic instability is based on the quasi-neutral particle model $^{13}$ which modifies the Poisson solver to take into account the importance of the near quasi-neutrality. In this model for electrons, we have only thermal adiabatic electrons $n_{e}=n_{0} \exp \left(e \varphi / T_{e}\right)$ in the massless limit, which rapidly cancels the charge separation due to the ion density fluctuations. By eliminating the electron dynamics we gain a large numerical advantage i.e., large time step satisfying $\omega_{p i} \Delta t<0.5$ instead of the condition $\omega_{p e} \Delta t<0.5$ to resolve high frequency electron plasma oscillations. In giving up the electron oscillations and parallel electron velocity distribution we loose a weak flattening of the very low velocity part of electron velocity distribution function. The adiabatic electron response appears in the Poisson solver and remains nonlinear. The ion dynamics determine the low irequency collective fluctuations with the ions advanced in time by the standard 1-D leap frog particle pusher. The field solver which supplies the electric force to the particle pusher is the 1-D iterative Poisson equation solver. Since the electron response has its intrinsic exponentially strong nonlinearity and is a source term in the Poisson equation several iterations of the Poisson equation solver are necessary in the nonlinear stage.

The iterative 1-D Poisson solver with Boltzman electron

$$
n_{e}=n_{0} \exp \left(\frac{\epsilon \varphi}{T_{e}}\right)
$$


is obtained by rewriting the charge density

$$
\frac{\partial^{2} \varphi}{\partial x^{2}}=-4 \pi e\left(n_{i}-n_{e}\right)=-4 \pi e\left[n_{i}-n_{0} \exp \left(\frac{e \varphi}{T_{e}}\right)\right]
$$

which is exactly rewritten as

$$
\frac{\partial^{2} \varphi}{\partial x^{2}}-\frac{4 \pi n_{0} e^{2}}{T_{e}} \varphi=-4 \pi e\left[n_{i}-n_{0} \exp \left(\frac{e \varphi}{T_{e}}\right)+n_{0}\left(1+\frac{e \varphi}{T_{e}}\right)-n_{0}\right] .
$$

By subtracting linearized Boltzman electron response from both sides of the equation and including the Debye shielding term in the left-hand side of Eq. (8) we can minimize the iteration at each time step required to solve Eq. (8). The final form of iteration scheme is

$$
\frac{\partial^{2} \varphi^{(n+1)}}{\partial x^{2}}-4 \pi n_{0} \frac{e^{2}}{T_{e}} \varphi^{(n+1)}=-4 \pi e\left[n_{i}^{(n)}-n_{0}\right]-4 \pi e n_{0}\left[\left\{1+\frac{e \varphi^{(n)}}{T_{e}}\right\}-\exp \left(\frac{e \varphi^{(n)}}{T_{e}}\right)\right]
$$

with boundary conditions $\varphi\left(x+L_{x}\right)=\varphi(x)$.

The reference simulation parameters, as given in Table II are the system length $L_{x}=$ $128 \Delta$, light ion stream velocity $v_{D L}=0.045 \Delta \omega_{p e}$, heavy ion stream velocity $v_{D H}=0.0$, particle size $a_{x}=1 . \Delta, m_{L} / m_{e}=1836, m_{H} / m_{e}=16 \times 1836$, initial temperature ratio $T_{e} / T_{i}=100$, total run time is $N \Delta t=4000 \times 10 \omega_{p e}^{-1}$, total number of particle is $n_{0}=$ $n_{0 L}+n_{0 H}=25600$.

\section{Qualitative Description of the Evolution of the Ion Acoustic Instability}

To see how the ion thermalization depends on the light to total ion density ratio $n_{0 L} / n_{0}$ a series of simulations are performed with other simulation parameters held fixed. The measured saturated heavy ion temperatures are shown in Fig. 7 along with the light ion temperatures for the fractional densities $n_{0 L} / n_{0}=1 / 2,2 / 3,3 / 4,4 / 5$. As $n_{0 L} / n_{0}$ becomes larger the heating ratio of the heavy ion temperature increases rapidly reaching $T_{H f} / T_{H i}=$ 29.4 at $n_{O L} / n_{0}=4 / 5$, where $T_{H f}$ is the final heavy ion temperature and $T_{H i}$ is the initial heavy ion temperature. In contrast the heating of the light ion temperature is essentially 
constant with $T_{L f} / T_{L i} \sim 12$. The final heavy ion temperature level $T_{H J} / T_{H i}$ for $n_{0 L} / n_{0}=4 / 5$ is about three times larger than that of $T_{H f} / T_{H i}$ for $n_{0 L} / n_{0}=1 / 2$. The difference in the time behavior of the thermalization in these two case is shown in Fig. 8 for the heavy ion temperature and in Fig. 9 for the light ion temperature.

To understand the saturation mechanism of the heavy ion temperature we look at the phase space $\left(x, v_{x}\right)$ evolution along with the plot of the spatial variation of electrostatic potential at several times of interest in the evolution. Five time values are selected in the heating sequence and shown in Figs. 10 and 11 for the $n_{0 L} / n_{0}=3 / 4$ case $(\eta=1 / 48)$. At $t=0$ the light and heavy ion streams are loaded with equal temperatures as shown in Fig. 10a,b. At $t \omega_{p e}=2000\left(t \omega_{p L}=47\right)$, in Fig. 10c which is in the linear stage, the phase space $\left(x, v_{x}\right)$ plot shows that both the light and heavy ion species are driven by the fastest growing linearly unstable $m=8$ mode with $k \lambda_{D e}=4.5$. In Fig. 10d, we show the spatial variation of $\exp \left(e \varphi / T_{e}\right)$ at the same time and see that at the position of the negative potential wells where the density has a depression there exists a corresponding cavity structure in phase space. At $t \omega_{p e}=4000\left(t \omega_{p L}=93\right)$, in Fig. $10 \mathrm{e}$, which is the beginning of nonlinear stage where linear $m=8$ mode coalesces to the $m=6$ mode. At $t \omega_{p e}=10,000\left(t \omega_{p L}=233\right)$, in Fig. $11 \mathrm{~g}$, which is in the middle of nonlinear heavy ion heating process, modes are coalesced into $m=3$ mode. At $t \omega_{p e}=40,000\left(t \omega_{p L}=934\right)$, in Fig. 11i, heavy ion temperature reaches its saturation level with $m=2$ coalesced mode. Comparing Fig. $11 \mathrm{~h}$ and Fig. $11 \mathrm{j}$ we see that the location of the coalesced phase space vortex has its cavity exactly matched with the electrostatic potential wells which are marked by arrow in Fig. 11h,j.

To have a closer look at the phase space evolution separate phase space diagrams for each ion species along with the corresponding spatial behavior of $e \varphi / T_{e}$ are made in Figs. 12, 13 and 14. At the linear stage $t \omega_{p e}=2000\left(t \omega_{p L}=47\right)$ (Fig. 12a) we see that both the light and heavy ion species are accelerated by the electrostatic field. A substantial fraction of the light ion species oscillate around the minima of the electrostatic potential. The ions cross 
the trapping separatrix as the waves grow in amplitude but are excluded from the interior of the vortex by the KAM surfaces that occur in the single particle Hamiltonian $H\left(p_{x}, x, t\right)$. From the sequence in Figs. 12, 13, and 14 one sees that the light ion species are strongly accelerated only during the linear regime. The heavy ions having ripples in their phase space stream with an amplitude comparable to its thermal velocity and oscillates with the same frequency as the light ion. At $t \omega_{p e}=3000\left(t \omega_{p L}=70\right)$ in Fig. $12 \mathrm{~b}$, which is in the beginning of nonlinear stage the amplitude of the ripple in the heavy ion stream becomes more than twice the value in its linear stage. The linear mode structure of the $m=8$ shown in both ion species is experiencing slight distortion. At $t \omega_{p e}=4000\left(t \omega_{p L}=93\right)$ in frame (c), which is at the middle of nonlinear heavy ion heating process, the mode with $m=8$ has coalesced to the mode $m=6$. The amplitude of the heavy ion ripple is now tripled and its linear mode structure begin to loose its shape. From $t \omega_{p e}=8000\left(t \omega_{p L}=187\right)$ in frame (d) to $t \omega_{p e}=40,000\left(t \omega_{p L}=934\right)$ in frame (f) the coalesced modes $(m=5 \sim 6)$ are marching to longer wavelength $(m=2 \sim 3)$ that are domination at the saturation stage. Both the light and heavy ion streams have lost their linear wave structure completely and the heavy ion species becomes thermalized uniformly over the phase space. The light ion species partially preserves their low $m$ mode structure with long-lived cavities in its phase space. In the high amplitude regime the light ion bounce or trapping frequency $\omega_{b L}=k\left(e \varphi / m_{L}\right)^{1 / 2}$ is faster than the growth rate $\gamma$, the phase space cavities are required by the KAM surfaces in the single particle Hamiltonian. The adiabatic invariance of the single particle action $J(H, t)$ given by

$$
J=\oint\left[\frac{2}{m}(H-e \varphi(x, t))\right]^{1 / 2} d x
$$

which prevents the ion from filling the cavities wher $\gamma \ll \omega_{b L}=k\left(e \varphi / m_{L}\right)^{1 / 2}$. All the phase space cavities shown in Figs. 12 and 13 are matched with the negative potential wells of $e_{\varphi} / T_{e}$ in Fig. 14. The depth of the potential well increases from the linear state to the nonlinear heating stage and then becomes shallow again in the slowly decaying state after 
saturation.

\section{Evolution of the Wavenumber Spectrum}

The time evolution of the average wavenumber $\langle m\rangle_{\text {ave }}$ in the spectrum is computed and shown here for the $n_{0 L} / n_{0}=4 / 5$ and $n_{0 L} / n_{0}=1 / 2$ cases. The evolution in Fig. 15 shows the rapid convergence of the average wavenumber to low-m number modes. The lowest average wavenumber is $\langle m\rangle_{\text {ave }}=2.5$ for both of the two different light ion concentration ratios. But the number of periodic cycles required to reach the lowest $\langle m\rangle$ number is larger for the $n_{0 L} / n_{0}=4 / 5$ case than for the $n_{0 L} / n_{0}=1 / 2$ case. For these cases the most unstable electrostatic mode is $m=9$ for $n_{0 L} / n_{0}=4 / 5$ and $m=7$ for $n_{0 L} / n_{0}=1 / 2$. The time evolution of the electrostatic field energy of the most unstable modes and also of the $m=2$ modes are given in Fig. 16. The fastest growing mode energy decays by two order of magnitude after trapping of the particle at the end of the linear growth. While the most unstable mode $m=9$ experiences strong damping, the lowest persistent mode, $m=2$ reaches a. quasi-steady state in both cases.

The frequency modulation appears during the field energy saturation. For the $n_{0 L} / n_{0}=$ $1 / 2$ case, the $m=2$ mode saturation level has weak damping and a weak modulation of the saturation level. The modulation with a period of about ten times the basic period is much stronger in the case of $n_{0 L} / n_{0}=4 / 5$. Figure 17 shows that the longest $m=1$ mode in the system is modulated by the short wavelength mode for the $n_{0 L} / n_{0}=4 / 5$ case and the $n_{0 L} / n_{0}=1 / 2$ case also shows evidence of wave modulation. These wave modulations are shown clearly by the two time correlation spectrum for the most unstable modes: the $m=9$ for $n_{0 L} / n_{0}=4 / 5$ and the $m=7$ for $n_{0 L} / n_{0}=1 / 2$ in Fig. 18. Beat wave formation are shown in both real and imaginary part of the two time correlation function.

The heavy ion heating mechanism appears to be the result of the trapping oscillation and the nonlinear modulational instability which generates a modulation frequency below 
the heavy ion bounce frequency. The heavy ion bounce frequency is less than the heavy ion plasma frequency during the nonlinear heavy ion heating process (Fig. 19). At saturation the wave dispersion $\omega(k)$ shows that the low $m$-number dominance of wave propagation and the dispersive structure of high $m$-mode due to nonlinear ion Landau damping (Fig. 20).

\section{Dynamics of the Thermalization Process}

The evolution of the ion-ion acoustic system divides into three stages. Here we analyze these stages in some detail. The reference case shown in Figs. 10-11 is used for some of the application of the formulas although most of the consideration given apply for all regimes with the beam parameter $\eta \ll 1$ which excludes the nearly pure heavy ion plasma.

\section{A. Linear Stage}

During the linear growth phase the classical beam-plasma instability dynamics occurs as modified by the presence of the electron dielectric function

$$
\varepsilon_{0}(k)=1+\frac{1}{k^{2} \lambda_{D e}^{2}}
$$

produced by the polarization of the thermalized electrons. The plasma polarization strongly reduces the strength of the long wavelength electric fields but has only a weak effect on the small scale modes $k \lambda_{D e}>1$. In the early phase of the subsonic regime $\left(c_{s L}>v_{D} \equiv\right.$ $\left.v_{D L}-v_{D H}\right)$ the reduction from $\varepsilon_{0}(k)$ is small since the fastest growing modes have $k \sim$ $\omega_{p L} / v_{D}>\omega_{p L} / c_{s L} \simeq \lambda_{p c}^{-1} ; c_{s l} \equiv\left(T_{e} / m_{i L}\right)^{1 / 2} \cong \lambda_{D e}^{-1}$. In addition to the dielectric shielding there is a weak dissipation produced by wave resonances with the very low velocity electrons giving

$$
\operatorname{Im} \varepsilon_{0}(k)=i\left(\frac{\pi}{2}\right)^{1 / 2}\left(k \lambda_{D e}\right)^{-2}\left(\frac{\omega}{|k| v_{e}}\right) \simeq\left(\frac{\pi}{2}\right)^{1 / 2}\left(\frac{m_{e}}{m_{i}}\right)^{1 / 2}\left(\frac{v_{D}}{c_{s}}\right)^{3}
$$

where we use $k \simeq \omega_{p L} / v_{D}$ for the unstatle spectrum. We completely neglect this weak dissipation of the waves into the electron distribution. Retaining the electron resonance 
would only produce a small flattening of the electron distribution $f_{e}(v)$ for $|v| \leq \omega / k<c_{s}$. In the supersonic regime $v_{D}>c_{s}$ this electron heating becomes appreciable.

In the early hydrodynamic beam-plasma phase of the system the evolution is controlled by the beam parameter through Eq. (6)

$$
\eta=\frac{n_{H}}{n_{L}} \frac{m_{L}}{m_{H}} \equiv \frac{n_{2}}{n_{1}} \frac{m_{1}}{m_{2}}
$$

For a heavy-light ion mixture the beam parameter is small unless the plasma has only a trace of the light ion density $n_{L} / n_{H} \leq m_{L} / m_{H} \ll 1$. We exclude this unusual case $(\eta>1)$ here.

The phase velocity at the critical condition for the ion-ion beam-plasma instability is given by

$$
\left(\frac{\omega}{k}\right)_{c}=\frac{v_{2}+\eta^{1 / 3} v_{1}}{1+\eta^{1 / 3}} \simeq v_{2}+\eta^{1 / 3}\left(v_{1}-v_{2}\right)
$$

which is determined by the critical condition $\partial \varepsilon / \partial \omega=0$ with Eq. (5) and is independent of the electron polarization $\varepsilon_{0}(k)$. For the critical beam plasma mode in Eq. (12) to be in the fluid regime the shift of the phase velocity from $v_{2}$ must be out of the thermal spread $v_{T 2}=\left(T_{2} / m_{2}\right)^{1 / 2}$ of the heavy ion species. This condition gives the threshold condition for the beam-plasma instability $\eta^{1 / 3} v_{D}>v_{T_{2}}=\left(T_{2} / m_{2}\right)^{1 / 2}$ where $v_{D}=\left|v_{1}-v_{2}\right|$. When this condition on $\eta>\left(v_{T_{2}} / v_{D}\right)^{3}$ is well satisfied the instability is in the hydrodynamic regime. As the thermal velocity spread increases in the heavy ion species so that $v_{T_{2}} / v_{D} \gtrsim \eta^{1 / 3}$ the instability goes into the slow-growing kinetic regime where the growth rate is determined by ¿f $/ \partial v$ at $v=\omega_{k} / k$.

The simulations start with ion distributions in the hydrodynamic regime $v_{T 2} / v_{D} \ll \eta^{1 / 3}$. In this regime the fastest growing mode $\left(k_{m}, \gamma_{m}\right)$ has a phase velocity closer to the speed of the heavy ions by approximately one half the difference of the velocity $\left(\omega / k-v_{2}\right)$ given in Eq. (12). Namely, the fastest growing mode has the speed

$$
\left(\frac{\omega}{k}\right)_{m} \cong v_{2}+\frac{1}{2}\left(\frac{\eta}{2}\right)^{1 / 3} v_{D}
$$


and the maximum growth rate is

$$
\gamma_{m}=\frac{\sqrt{3}}{2}\left(\frac{\eta}{2}\right)^{q / 3} \omega_{p 1}\left(1-v_{D}^{2} / c_{s L}^{2}\right)^{1 / 2}
$$

a.t the wavenumber

$$
k_{r n}=\frac{\omega_{p 1}}{v_{D}}\left(1-v_{D}^{2} / c_{A L}^{2}\right)^{1 / 2} .
$$

Equations (13)-(15) are the well-known beam-plasma equations ${ }^{14}$ applied to two ion species (heavy and light) streaming system. Modes with $k \ll k_{m}$ are also unstable but have a different physical origin. For the nonresonant modes $k<k_{m}$ the light ion plasma provides a negative dielectric response $\varepsilon_{1}(k, \omega) \equiv \omega_{0}(k)-\omega_{p 1}^{2} / \omega^{2}<0$ along the resonance $\omega=k v_{D}$. Thus, the heavy ion plasma oscillations at $\omega_{p H}$ become uristable with

$$
\omega=k v_{2} \pm i \frac{\eta^{2 / 3} \omega_{p 1}}{V-\varepsilon_{1}\left(k, k v_{D}\right)}
$$

In $\mathrm{Eq} .(16) \varepsilon_{1}<0$ for $k<k_{m}$ given in $\mathrm{Eq}$. (15). The resonant mode $k_{m}$ with the maximum growth given in Eqs. (13)-(15) occurs in the limit $\varepsilon_{1}\left(k, k v_{D}\right) \rightarrow 0$ in Eq. (16).

For the reference case shown in Figs, 10-11 for the hydrogen-oxygen mixture $\left(n_{1} / n_{0}=\right.$ $\left.3 / 4, n_{2} / n_{0}=1 / 4\right)$ the beam parameter is $\eta=1 / 48$ and the expansion parameter is $\eta^{1 / 3}=$ 0.276 . The hydrodynamic condition is well satisfied since $v_{D}=0.045 \Delta \omega_{p e}=c_{u} L_{1} / 2$ and $v_{T_{i}}=1 / 4 v_{T_{1}} \cong 0.003 \Delta \omega_{p e}$. In the phase space plots the rest frame is chosen as the oxygen drift frame so that $v_{2}=0$ and $v_{1}=v_{D}=0.045 \Delta \omega_{\text {pe }}$.

For the relerence case in Fig. 10 the phase velocity of the critical mode fron Eq. (12) is $(\omega / k)_{c}=0.216 v_{D}=0.0097 \Delta \omega_{\text {pee }}$. The fastest growing mode according to Eqs. (13)-(1.5) has $k_{m}=\left(\omega_{p 1} / v_{D}\right)\left(1-v_{D}^{2} / c_{s}^{2}\right)^{1 / 2}=\sqrt{3} \omega_{p 1} / c_{0}$ corresponding to $k \lambda_{D_{e}}=1.5$ or using $k \Delta=$ $2 \pi m \Delta / L_{x}$ to $m=8$ in the periodic simulation box. In the hydrodynamic approxirnation the growth rate is $\gamma / \omega_{p 1}=\left(\frac{\sqrt{3}}{2}\right)^{2}(0.219)=0.164$ where $\omega_{p 1}=\omega_{p e}\left(n_{1} m_{e} / n_{0} m_{1}\right)^{1 / 2}=0 .\left(1202 \omega_{p e}\right.$.

In Fig. 10 the evolution of the two-ion streams in the phase space is shown. Frames (a) and (b) at $t=0$ shows the initial Maxwellians in the oxygen rest frame $\left(v_{2}=0\right)$ with the 
drift velocity $v_{D}=0.5 c_{s}=0.045 \Delta \omega_{p e}$. Frame (c) at $t \omega_{p e}=2000\left(t \omega_{p L}=47\right)$ shows the begirning of the trapping of the light ions which ends the exponential growth of the beam mode. Frame (e) at $t \omega_{p e}=4000\left(t \omega_{p L}=94\right)$ shows the trapping of the heavy ions and the thermalization of the light ions.

\section{B. Trapping Phase of the Evolution}

The linear growth rate of the beam mode continues from the initial noise level until the resonant ion orbits cross the separatrix becoming trapped in the negative potential wells of $\varphi(x, t)$. Here we calculate the trapping amplitudes for the light and heavy ions.

In the wave frame $v_{\phi}=\omega / k$ the single ion Hamiltonians are

$$
H=\frac{1}{2} m_{\alpha}\left(v^{\prime}\right)^{2}+e_{\alpha} \varphi(t) \cos (k x)
$$

where $v^{\prime}=v_{\alpha}-v_{\varphi}$ and $\alpha$ stands for ion species. Neglecting the initial thermal spreads, the typical ion velocities are taken as $v_{1}$ and $v_{2}$ and the trapping condition for Hamiltonian Eq. (17) is $H=e_{a} \varphi$ giving for the light ions the critical wave amplitude

$$
e \varphi_{1}^{t r}=\frac{1}{2} m_{1}\left(v_{1}-v_{\phi}\right)^{2} \cong \frac{m_{1} v_{D}^{2} / 2}{\left(1+\eta^{1 / 3}\right)^{2}}
$$

and for the heavy ions

$$
e \varphi_{2}^{t r}=\frac{1}{2} m_{2}\left(v_{2}-v_{\phi}\right)^{2} \cong \frac{m_{2} \eta^{2 / 3} v_{D}^{2} / 2}{\left(1+\eta^{1 / 3}\right)^{2}}
$$

where we use the critical phase velocity in Eq. (12) to calculate the quantities $\left(v_{\alpha}-v_{\phi}\right)^{2}$. Using the phase velocity from Eq. (13) rather than Eq. (12) lowers the trapping amplitude for the heavy ions to $e \varphi_{2}^{t r} \simeq 0.2 m_{2} \eta^{2 / 3} v_{D}^{2}$. The phase velocity $v_{\phi}$ inferred from the center of the trapping vortices in Fig. $10 \mathrm{c}$ is $v_{\phi} \simeq 0.01 \Delta \omega_{\text {pe }}$ which is in better agreement with the value given in Eq. (12) $\left(\eta^{1 / 3} v_{D}=0.0115 \Delta \omega_{p e}\right)$ than the value in Eq. (13).

Comparing the trapping conditions in Eqs. (18) and (1.9) we see that the light ions are trapped first $\left(e_{1} \psi_{1}^{t r}<e_{2} \varphi_{2}^{t r}\right)$ unless the beam parameter $\eta$ is very small. Comparing $\psi_{1}^{t r}$ and 
$\varphi_{2}^{\text {tr }}$ in Eqs. (18) and (19) we see that for sufficiently small $\eta$ given by

$$
\eta<\eta_{2} \equiv\left(\frac{m_{1}}{m_{2}}\right)^{3 / 2}
$$

which requires the low concentration of heavy ions

$$
\left(\frac{n_{2}}{n_{1}}\right)<\left(\frac{m_{1}}{m_{2}}\right)^{1 / 2}
$$

When the low concentration of heavy ions given by Eq. (21) is satisfied the phase velocity is sufficiently close to the heavy ion streaming velocity that the heavy ions are trapped first and we expect that the heavy ions are thermalized more effectively since they provided the limit to the beam mode growth beiore the light ions are trapped. In the case where $n_{2} / n_{1}=1 / 4$ and $\left(m_{1} / m_{2}\right)^{1 / 2}=1 / 4$ the trapping of the heavy and the light ions occur near the same amplitude. The phase space diagrams show that the tail of the light ions are trapped just prior to the heavy ion trapping indicating that the neglect of the light ion thermal velocity spread in Eqs. (18) and (19) is not well satisfied in this particular case. Now we consider the parameters for this case in more detail.

In the reference case in which $n_{2} / n_{1}=1 / 4$ and $\left(m_{1} / m_{2}\right)^{1 / 2}=1 / 4$ the trapping conditions are now from Eqs. (18) and (19). These amplitudes are comparable to the maximum amplitudes shown in Fig. 14, where $\left|e \varphi_{\max } / T_{e}\right| \simeq 0.06$ in frarne (a) and $\left|e \varphi_{\max } / T_{e}\right| \simeq 0.08-0.09$ in frame (b). These frames correspond to the phase space plots in frames (a) and (b) in Fig. 12 where the apparent phase velocity is $\left|v_{\phi}\right| \simeq 0.06 \$ \Delta \omega_{p e}=1.44 v_{D}$. Using Eq. (12) with $v_{2}=0, v_{1}=0.045 \Delta \omega_{p e}, \eta^{1 / 3}=0.217$ and $c_{s L}=0.093 \Delta \omega_{p e}$ we compute the dirnensionless trapping amplitude

$$
\frac{e \varphi_{1}^{t r}}{T_{e}}=\frac{1}{2}\left(\frac{.0353}{.0933}\right)^{2}=0.071
$$

and

$$
\frac{e \varphi_{i}^{t r}}{T_{e}}=\frac{1}{2}\left(\frac{.005}{.093}\right)^{2}=0.08
$$


from Eqs. (18) and (19). Thus, for this case the light and heavy ions are trapped at comparable amplitudes although the light are still presumably trapped. This case $n_{2} / n_{1}=1 / 4$ has the strongest heavy ion heating.

\section{Vortex Coalescence in Phase Space}

At the time $t \omega_{p e}=4000\left(t \omega_{p L}=93\right)$ shown in frame (c) of Fig. 12 there is the beginning of the coalescence of the phase space trappings. Comparing frame (b) through (c) in Fig. 12 we see that while (b) has eight nearly equal amplitude trapping cells corresponding to the beam mode $k_{m}$ value by the time $t \omega_{p e}=4000$ in frame (c) there are six large trapping cells and two smaller cells beginning to disappear. The process continues to where at the stage in frame (d) of Fig. 13 there remains four large trapping cells. The result of this evaluation is a period doubling in the spatial domain and an approximate doubling in the time domain. At later times this period doubling process continues until there exist two large cells shown in frame (f) of Fig. 13.

In terms of fluid theory of the nonlinear waves the period doubling can be understood as the modulational instability producing the exponential growth of the mode $k_{0} / 2$ driven by the saturated large amplitude beam mode at $k_{0} \simeq k_{m}$.

At the end of the trapping stage, as in frame (c), there is a well-defined large amplitude wave

$$
\varphi=\varphi_{0} \cos \left(k_{0} x-\omega_{0} t\right)=\operatorname{Re}\left(\varphi_{0} e^{i k_{0} x-i \omega_{0} t}\right) .
$$

This large amplitude wave acts as a pump wave inducing periodic modulation for any longer wavelength $k<k_{0}$ plasma mode given locally by dispersion relation $\varepsilon\left(k, \omega \mid n_{k_{0}}(x, t)\right)=0$. This system with a modulation of the density is generically susceptible to the modulational or decay instability with $k_{0} \rightarrow k+k$ with an exponential growth of the $k=k_{0} / 2$ decay mode. In this stage of evolution the velocity distribution function is complicated so that $\varepsilon(k, \omega)$ has several branches as in Fig. Ia, but no strongly growing mode as appeared in Fig. 1 b since the 
thermalization of the beam has occurred to eliminate the linear driver. From the spectral distribution $I(k, \omega)$ in Fig. 20

$$
I(k, \omega)=\int_{-T / 2}^{T / 2} d \tau e^{i \omega \tau} \int_{t-T}^{t} \frac{d t}{T} E_{k}^{*}(t) E_{k}(t+\tau) .
$$

computed from $E_{k}(t)$ throughout the simulation run we find that the intensity of $I(k, \omega)$ is strongest along $\omega^{2} \simeq k^{2} v_{\phi}^{2}$ with $k \lambda_{D e} \lesssim 1 / 2$ and the wave speed $v_{\phi}$ is very near to $c_{s}$. The dominance of these long wavelength modes occurs in the late time stages. The long waves dispersion is given by

$$
\omega^{2}=k^{2} v_{\phi}^{2}\left[1-\frac{k^{2} v_{\phi}^{2}}{\omega_{p L}^{2}}\left(1-\frac{\delta n}{n}\right)\right]
$$

where $\delta n / n$ is the density modulation produced by the beam mode $k_{0}=k_{m}$. Taking $k=k_{0} / 2$ and defining the frequency mismatch $\delta \omega=2 \omega_{k}-\omega_{k_{0}}$ for the decay wave the stability of the decay wave $\varphi_{k}(t)$ is given by the Mathieu equation with

$$
\frac{d^{2} \varphi_{k}}{d t^{2}}+\omega_{k}^{2}\left(1+h \cos \omega_{0} t\right) \varphi_{k}=0
$$

with $h \cong\left(k^{2} \varphi^{2} / \omega_{p L}^{2}\right)(\delta n / 2 n)$. The decay wave varies as $\varphi_{k}(t)=e^{s t} \cos \left(\omega_{k_{0}} t / 2\right)$ with $s=$ $\pm\left(\omega_{k_{0}} / 2\right)\left(h^{2} / 4-\delta \omega^{2} / \omega_{k_{0}}^{2}\right)^{1 / 2}$ giving unstable growth for $|h|>2|\delta \omega| / \omega_{k_{0}}$ where $\delta \omega$ is the frequency mismatch between the modulational wave $k_{0}, \omega_{k_{0}}$ and the decay wave $\omega_{k}, k=k_{0} / 2$. We estimate that $|\delta \omega| / \omega_{k_{0}}$ is small, of order $k^{2} \lambda_{D e}^{2}$, so that the trapping potentials in Eqs. (17) and (18) give sufficiently large density modulations to be unstable to the growth of the period doubling decay modes.

\section{Conclusions}

For the case of weak or sub-sonic ion-ion acoustic one dimensional streaming turbulence considered here we find that the collective heating and subsequent evolution is already complicated with several distinct phases. The effective free energy from the relative stream velocity $v_{D}$ that goes into the waves at the initial wave breaking point is determined by the 
light ion streaming relative to the wave frame $\Delta E_{1}=\frac{1}{2} m_{1} v_{D}^{2} /\left(1+\eta^{1 / 3}\right)^{2}$ unless the concentration of heavy ions is very low $n_{2} / n_{1} \ll\left(m_{1} / m_{2}\right)^{1 / 2}$. For very low concentrations of heavy ions the situation changes as described in Sec. III.B. After the wave breaking, the light ions are thermalized with a rather flat velocity distribution that has a total energy content of order $m_{L} v_{D}^{2}$. The heavy ions develops low density tail of higher velocity particles. In this stage initial fast growing mode with wavenumber comparable to $\omega_{p L} / v_{D}$ are damped. The new wave-particle system shows a parametric decay to wavelength doubling. The dominant potential structure is transformed to the $m=2$ or 3 from the linear driving mode $m=7$ or 9 modes. The final state in which the total turbulent electrostatic potential energy has subsided to one half of its peak value is best characterized as a self-organized state with one or two phase space holes. Thus the evolution is to a final state which still has some free energy in that it is far from the Maxwellian velocity distributions. The final states are stable to the linear waves for the corresponding spatially averaged velocity $f_{1}(v)$ and $f_{2}(v)$ distributions as shown in Fig. 21. Nonetheless the system being out of equilibrium supports nonlinear self-organized collective modes of the form phase space holes as originally conjectured by Dupree. ${ }^{12}$ Thus, non-wave constituents are found to be important for describing the final state of the system.

\section{Acknowledgment}

This work was supported by the U.S. Department of Energy contract \#DE-FG05-80E'T53088 . 


\section{References}

1. R.L. Kaufmann et al., J. Geophys. Res. 91, 10080 (1986).

2. P.H. Reiff et al., Geophys. Monogr. Ser. 38, p83, AGU, Washington, D.C. (1986).

3. R. Bergmann, W. Lotko, J. Geophys. Res. 91, 7033 (1986).

4. P.B. Dusenbery and R.F. Martin, J. Geophys. Res. 92, 3261 (1987).

5. P.B. Dusenbery, R.F. Martin, and R.M. Winglee, J. Geıphys. Res. 93, 5655 (1988).

6. C.T. Dum, J. Geophys. Res. 94, 5655 (1989).

7. R.M. Winglee, P.B. Dusenbery, H.L. Collin, C.S. Lin, and A.M. Persson, J. Geophys. Res. 94, 8843 (1989).

8. D. Schriver, M. Ashour-Abdalla, H. Collin, and N. Lallande, J. Geophys. Res. 95, 1015 (1990).

9. D. Schriver and M. Ashour-Abdalla, J. Geophys. Res. 95, 3987 (1990).

10. M. W. Chen and M. Ashour-Abdalla, J. Geophys. Res. 95,18949 (1990).

11. H. Karimabadi, N. Omidi and K.B. Quest, Geophys. Res. Lett. 18, 1813, (1991).

12. T.H. Dupree, Phys. Fluids 25, 277 (1982).

13. H. Okuda, J.M. Dawson, A.T. Lin, and C.C. Lin, Phys. Fluids 21, 476 (1978).

14. A.B. Mikhailovskii, "Theory of Plasma Instabilities," Vol. I, p. 68, Consultant Bureau, (1973). 


\section{Figure Captions}

1. The four principal roots of the Vlasov dispersion relation $\mathrm{Eq}$. (1) for the reference parameters in Table I. (a) Mode frequencies $\omega / \omega_{p L}$ versus $k \lambda_{D e}$. (b) Mode growth rate $\gamma / \omega_{p L}$ versus $k \lambda_{D_{e}}$.

2. The four modes from the fluid dispersion relation Eq. (5). Labelled I, II, III and IV to correspond with Fig. 1. (a) mode frequency versus $k \lambda_{D e}$ and (b) mode growth rate versus $k \lambda_{D e}$

3. Comparison of the principal unstable mode I from Figs. 1 and 2. (a) mode frequency and (b) mode growth rates.

4. Variation with mixture ratio $n_{L} / n_{0}$ of the unstable mode I of (a) mode frequency and (b) groi th rate for the wavenumber $k \lambda_{D_{e}}=1.66$ which corresponds to $\gamma_{\max }$ in Fig. 3 .

5. Variation with heavy ion mass $m_{i H} / m_{e}$ of the unstable mode I (a) frequency and (b) growth rate at $k \lambda_{D e}=1.66$ and $n_{L}=n_{H}$.

6. Parameterization of the logarithmic dependence of the growth rate in Fig. 5 with the variation $m_{e} / m_{H}=1 / 1836$.

7. Variation of the measured saturated temperatures of heavy and light ions, solid triangle represents final heavy ion temperature $T_{H}\left(t_{s}\right) / T_{H}(0)$ and empiy square represents final light ion temperature $T_{L}\left(t_{s}\right) / T_{L}(0)$ as a function of the light ion fraction $n_{L} / n_{0}$.

8. Comparison of the heavy ion heating curves for the cases $(a)$ of $n_{L} / n_{0}=4 / 5$ and (b) $n_{L} / n_{0}=1 / 2$.

9. Comparison of the light ion heating curves for the cases (a) of $n_{L} / n_{0}=4 / 5$ and (b) $n_{L} / n_{0}=1 / 2$. 
10. and 11. Phase space dynamics of the unstable system for $n_{L} / n_{0}=3 / 4$. Here $\eta=1 / 48$ $\left(\eta^{1 / 3}=0.276\right)$.

(a), (b) $t \omega_{p e}=0$ initial configuration

(c), (d) $t \omega_{p e}=2 \times 10^{3}\left(t \omega_{p L}=47\right)$ near maximum of linear growth phase

((e), (f) $t \omega_{p e}=4 \times 10^{3}\left(t \omega_{p L}=93\right)$ beginning of nonlinear ion trapping occurred with a trapping potential $e \varphi / T_{e}$.

(g), (h) $t \omega_{p e}=10^{4}\left(t \omega_{p L}=233\right)$ coalescence of trapping oscillations into two phase space holes

and

(i), (j) $t \omega_{p e}=4 \times 10^{5}\left(t \omega_{p L}=934\right)$ final relaxed, stable state of the interaction of hydrogen/oxygen mixture

12. and 13. Phase space dynamics of the unstable system for $n_{L} / n_{0}=1 / 2$. Here $\eta=1 / 16$ and $\eta^{1 / 3}=0.397$

(a) $t \omega_{p e}=2 \times 10^{3}\left(t \omega_{p L}=47\right)$ at the peak of linear stage.

(b) $t \omega_{p e}=3 \times 10^{3}\left(t \omega_{p L}=70\right)$ At the beginning of nonlinear stage.

(c) $t \omega_{p e}=4 \times 10^{3}\left(t \omega_{p L}=93\right)$ At the middle of nonlinear heavy ion heating process.

(d), (e), and (f) correspond to $t \omega_{p e}=8 \times 10^{3}\left(t \omega_{p L}=187\right), t \omega_{p e}=10^{4}\left(t \omega_{p L}=233\right)$, and $t \omega_{p e}=4 \times 10^{4}\left(t \omega_{p L}=934\right)$ mode coalescence procedure at the saturation.

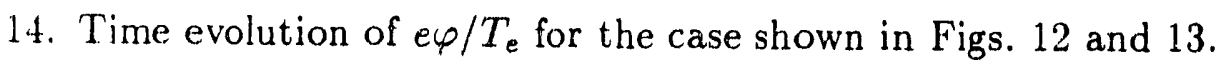

15. Time evolution of the mean wavenumber $\langle k(t)\rangle$ relative to the longest wavenumber $k_{\min }=2 \pi / L_{x}=0.012 \lambda_{D e}^{-1}$ in the box. (a) small heavy ion component $n_{L} / n_{0}=4 / 5$ and (b) equal heavy-light ion mixture $n_{L} / n_{0}=1 / 2$. 
16. Electrostatic field energy $\left\langle E_{k}^{2}(t)\right\rangle / 4 \pi n_{0} T_{e}$ for the fastest growing modes and the long wavelength $(m=2)$ modes. (a) and (b) for $n_{L} / n_{0}=4 / 5$ and $n_{L} / n_{0}=1 / 2$.

17. Time dependence of the autocorrelation function (a) small heavy ion component $n_{L} / n_{0}=$ $4 / 5$ and (b) equal heavy-light ion mixture $n_{L} / n_{0}=1 / 2$.

18. Time dependence of the autocorrelation function for $E_{k}(t)$ for most unstable modes (a) small heavy ion component $n_{L} / n_{0}=4 / 5$ and (b) equal heavy-light ion mixture $n_{L} / n_{0}=1 / 2$.

19. Time evolution of the ion temperature and the heavy ion bounce frequency for the case

20. Spectral distribution $I(k, \omega)$ from the $E_{k}(t)$ (a) for $n_{L} / n_{0}=4 / 5$ and (b) for equal heavy-light ion mixture $n_{L} / n_{0}=1 / 2$.

21. Heavy and light ion velocity distribution functions in the final relaxed state. 
Table I - Reference Plasma Parameters

Oxygen-Hydrogen Mixture

$$
\begin{aligned}
& m_{H} / m_{L}=m_{2} / m_{1}=16 \\
& n_{H} / n_{0}=n_{L} / n_{0}=0.5 \\
& \eta=\frac{n_{H} m_{L}}{n_{L} r_{H}}=\frac{1}{16} \\
& v_{D L}=0.045 \Delta \omega_{p e}=0.01125 v_{e} \\
& \quad=0.48 c_{s L} \\
& T_{i L}=T_{i H}=0.01 T_{e}
\end{aligned}
$$

Table II - Reference Simulation Parameters

$$
\begin{array}{ll}
\lambda_{D e}=4 \Delta & v_{T e}=4 \Delta \omega_{p e} \\
a=\Delta(\text { Particle Size }) & N_{H}=N_{L}=12800 \\
L_{x}=128 \Delta=512 \lambda_{D e} & \\
k_{\min }=2 \pi / L_{x}=0.012 \lambda_{\overline{D e}}^{-1} & \\
k_{\max }=\pi / \Delta=12.6 \lambda_{D e}^{-1} &
\end{array}
$$




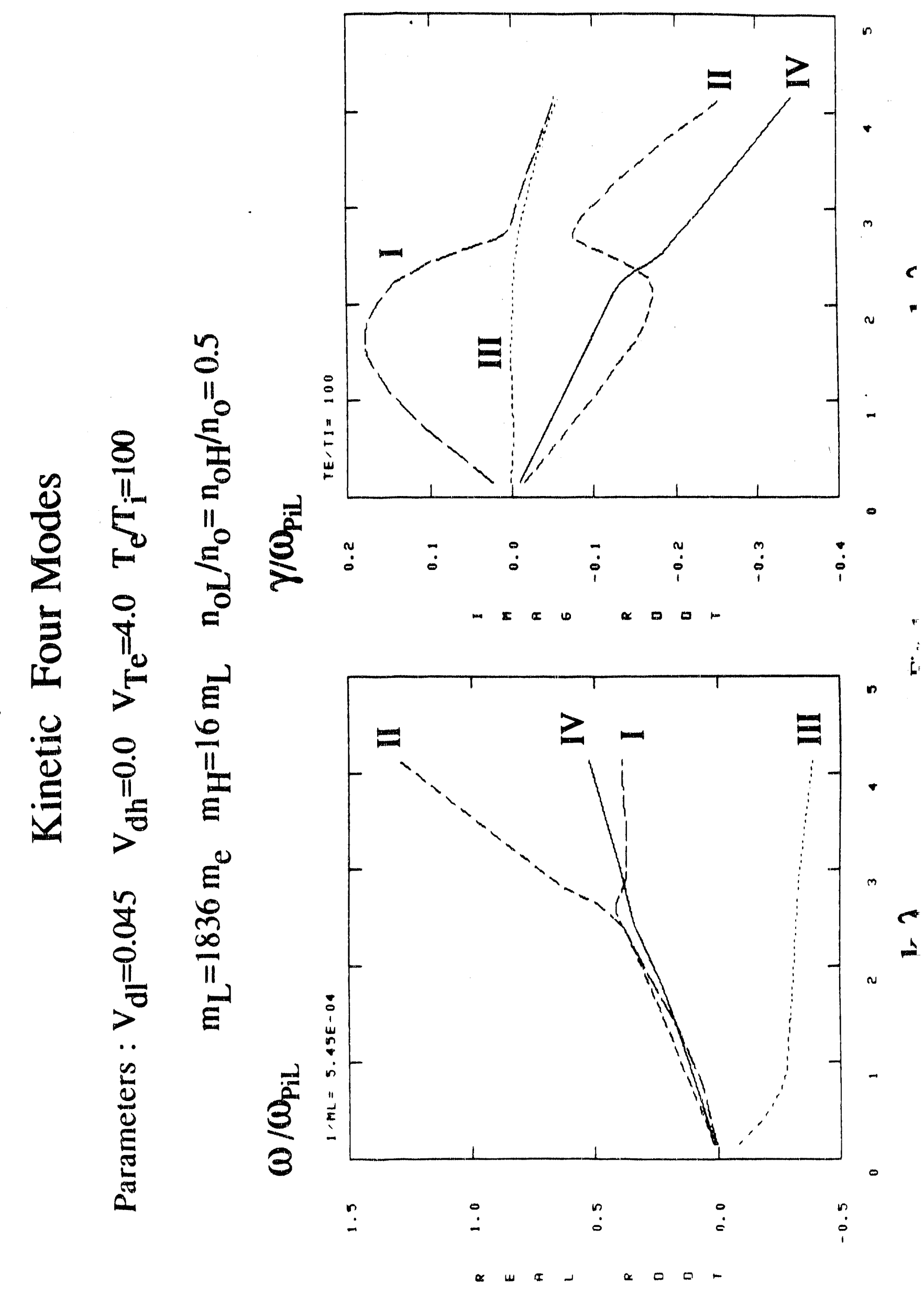




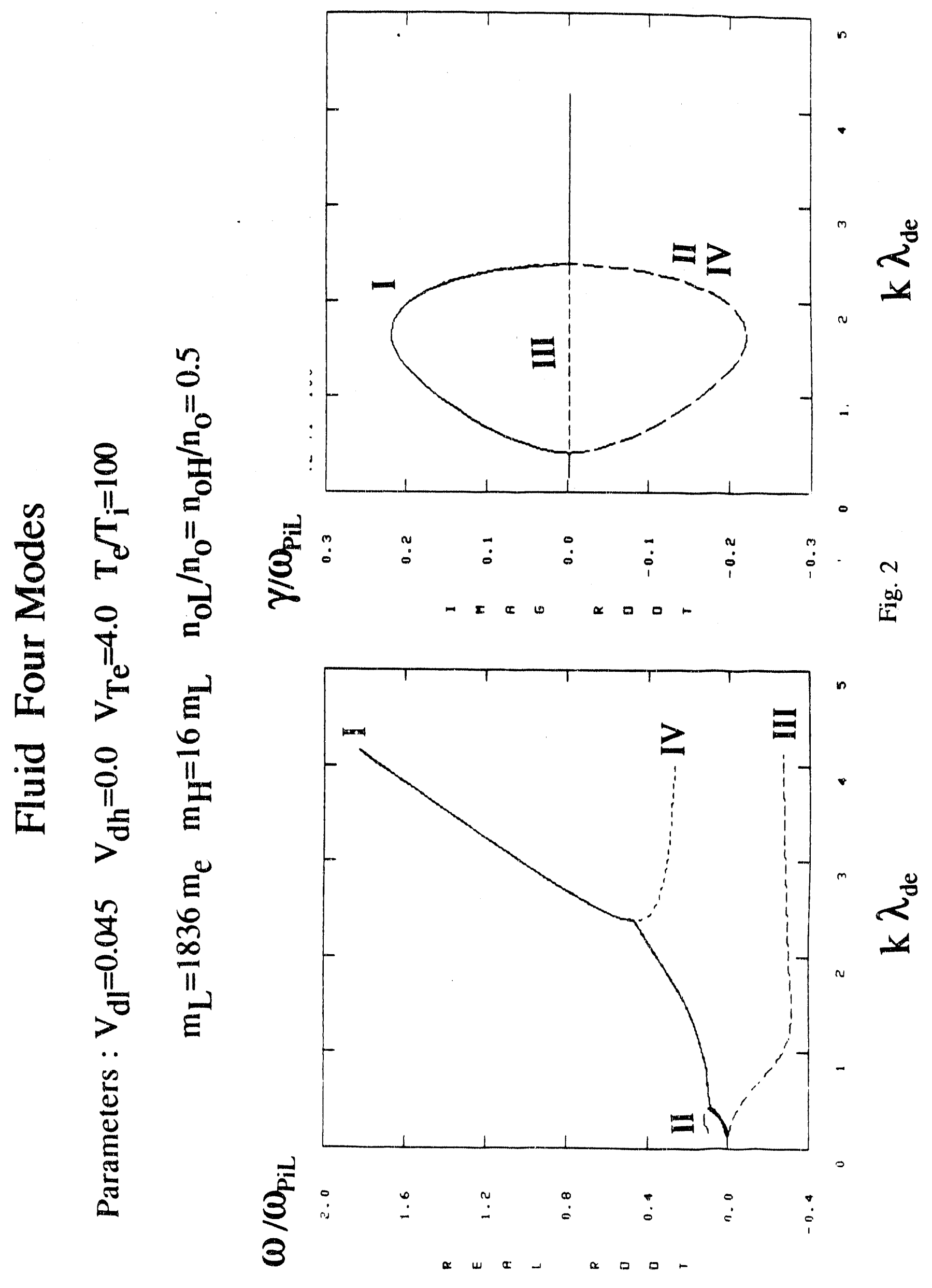



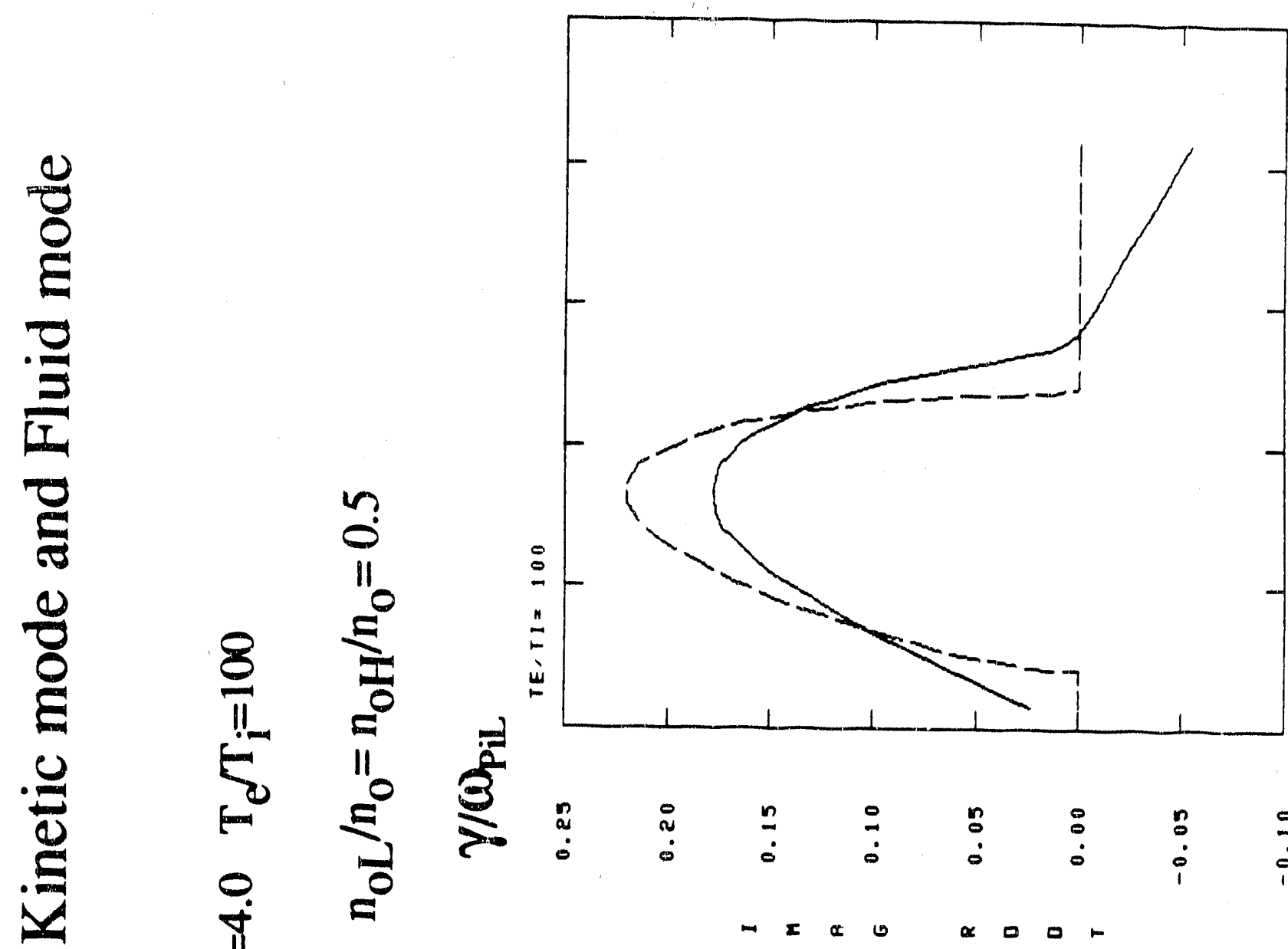

$\underset{\varrho}{\varrho}$

$\frac{\frac{0}{0}}{5}$

$-11$
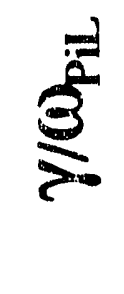

$\stackrel{n}{a} \quad \stackrel{0}{0}$

¿.

$\stackrel{n}{0}$

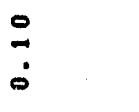

$\begin{array}{llll}n & : & n & 0 \\ 0 & 0 & \dot{0} & \dot{0}\end{array}$

- $\quad \& 0 \propto 001$

$\frac{8}{8}$

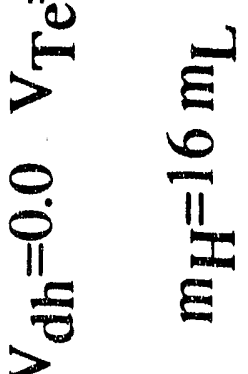

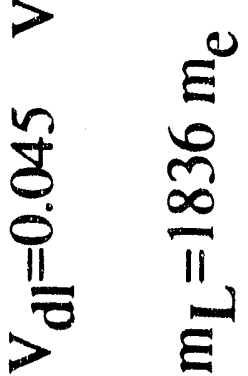
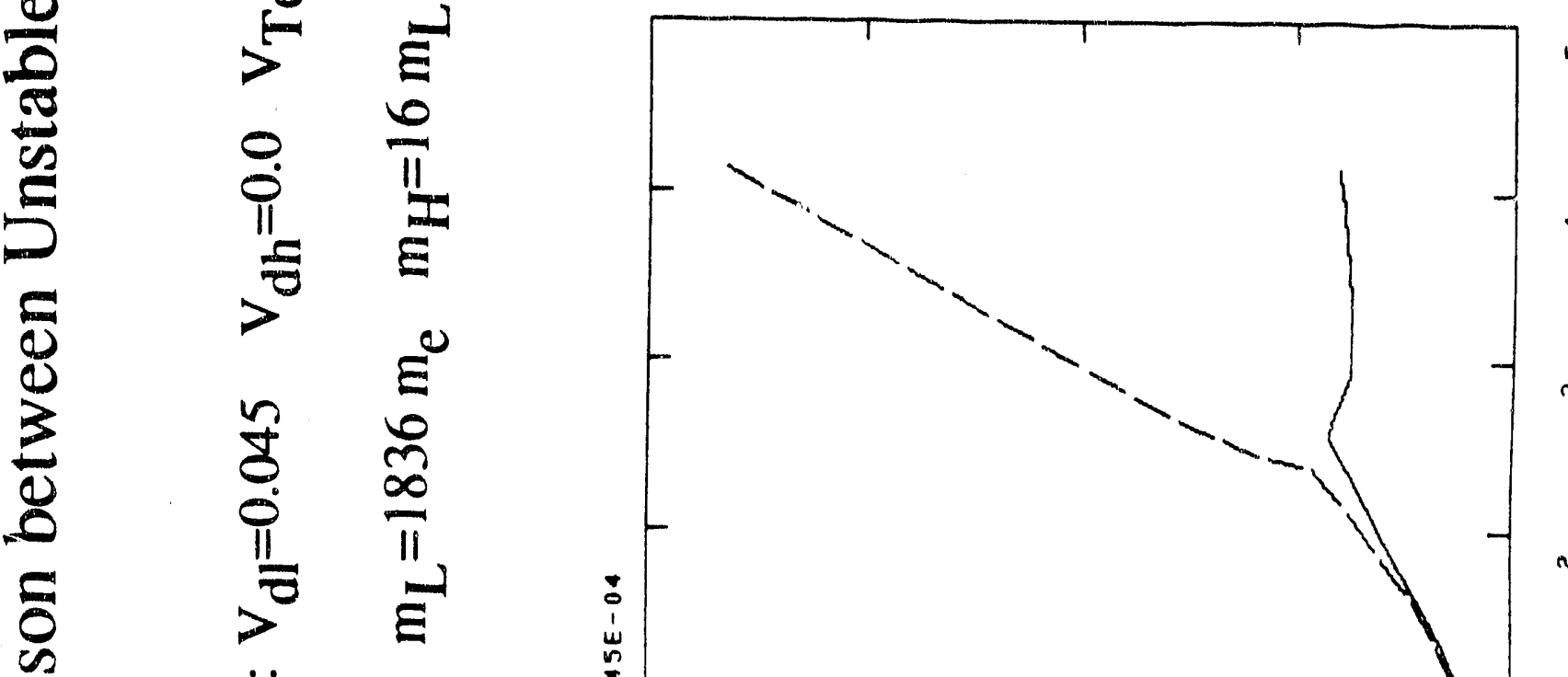

茪
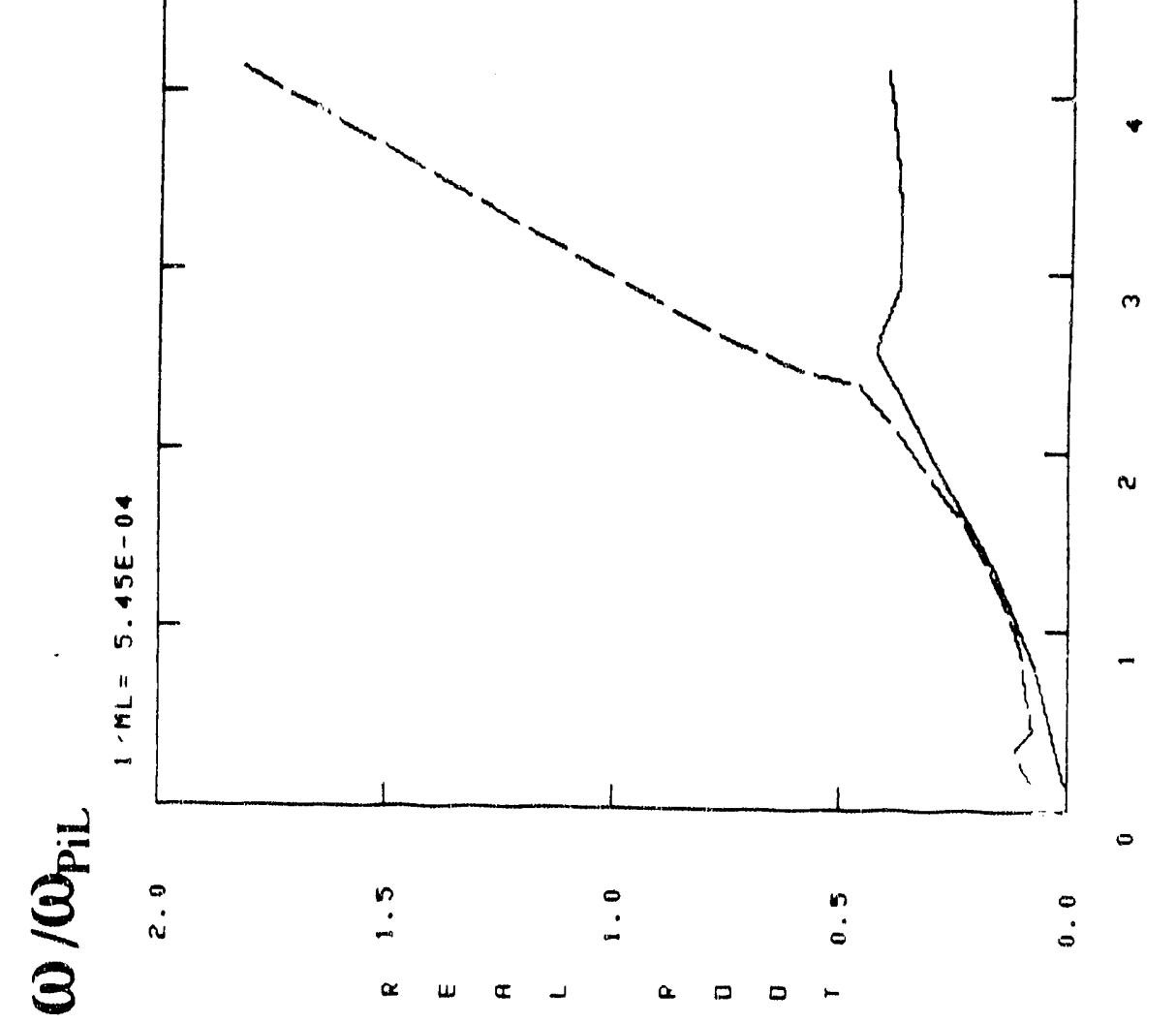


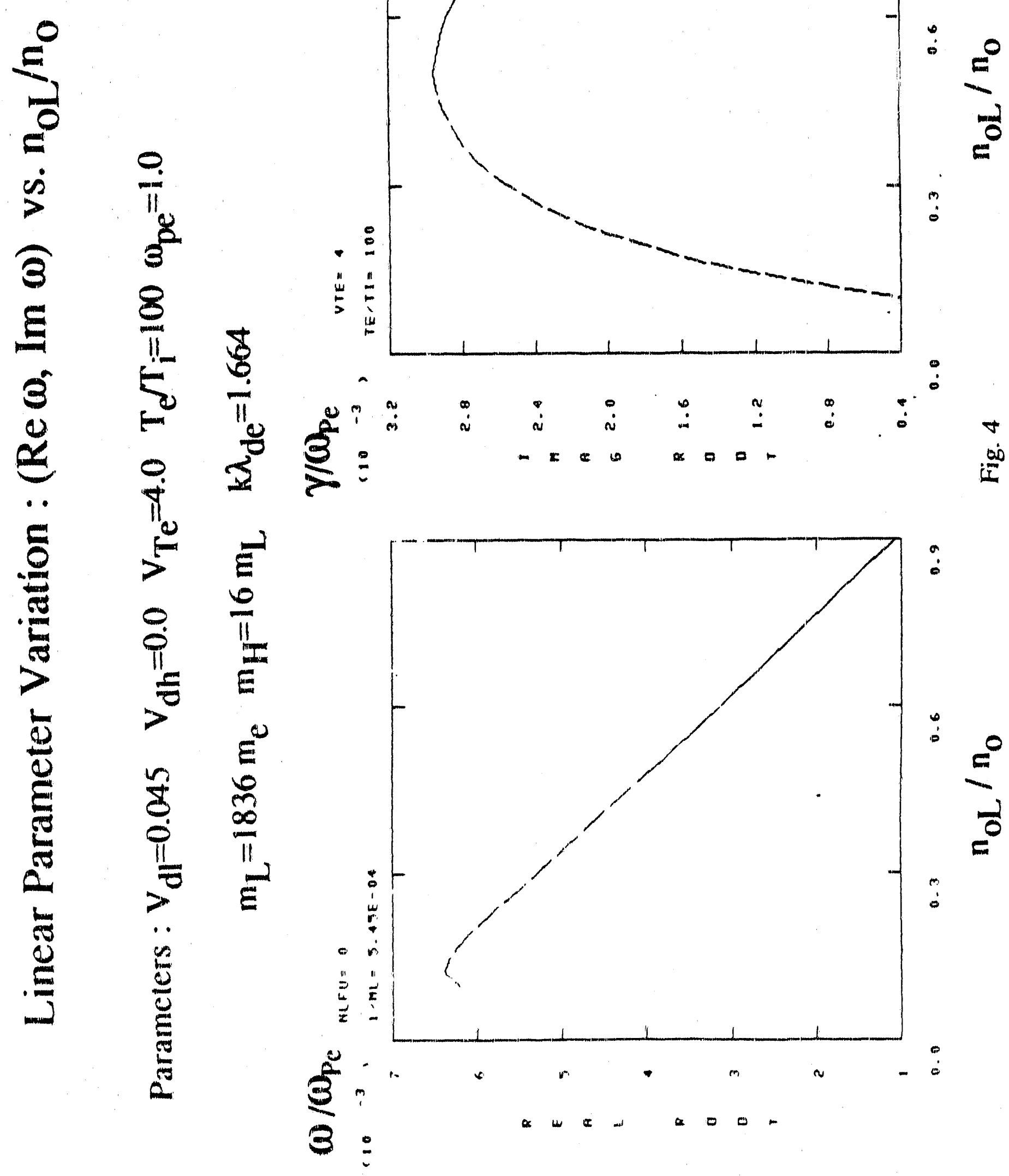



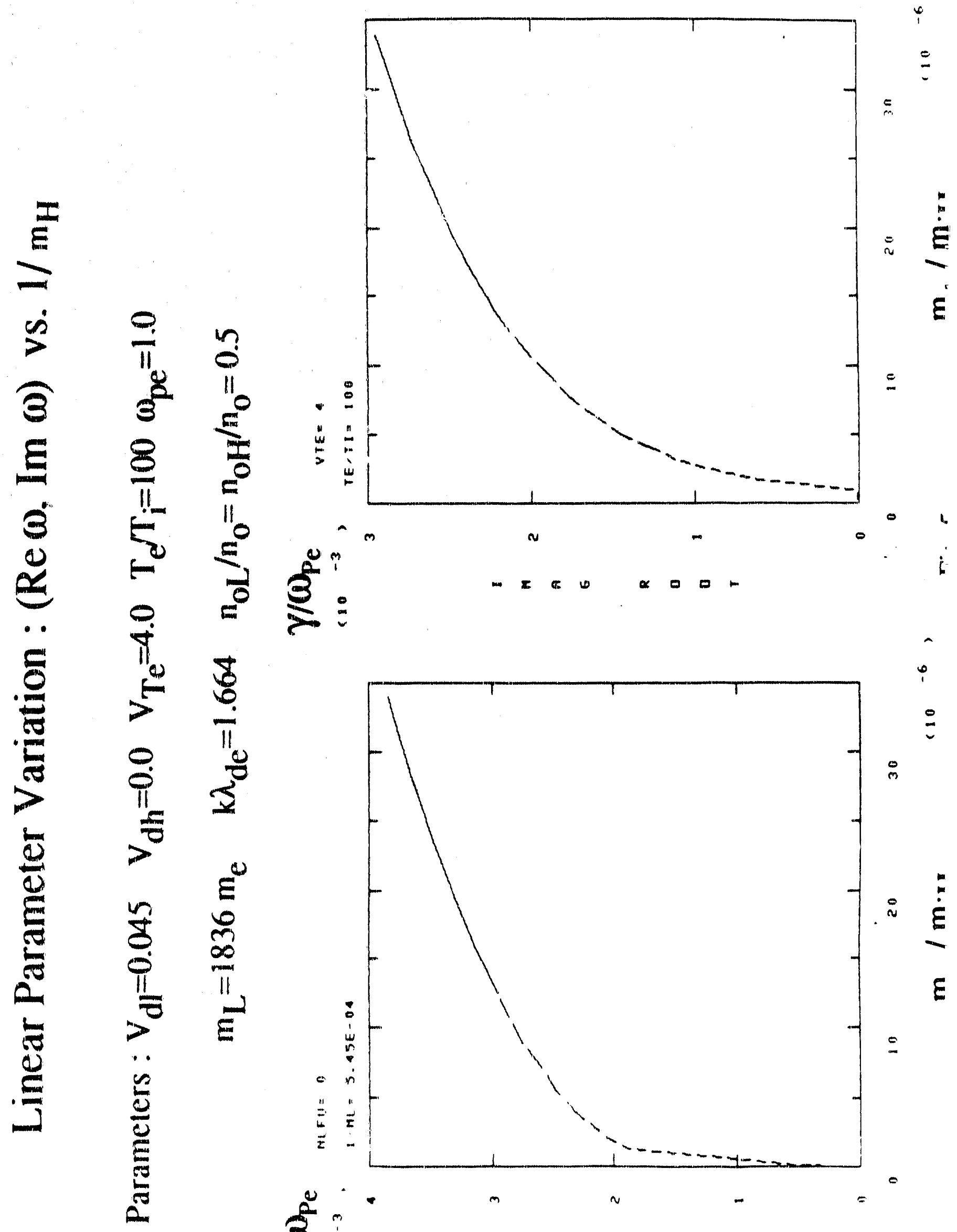

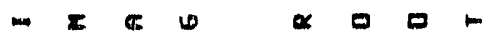

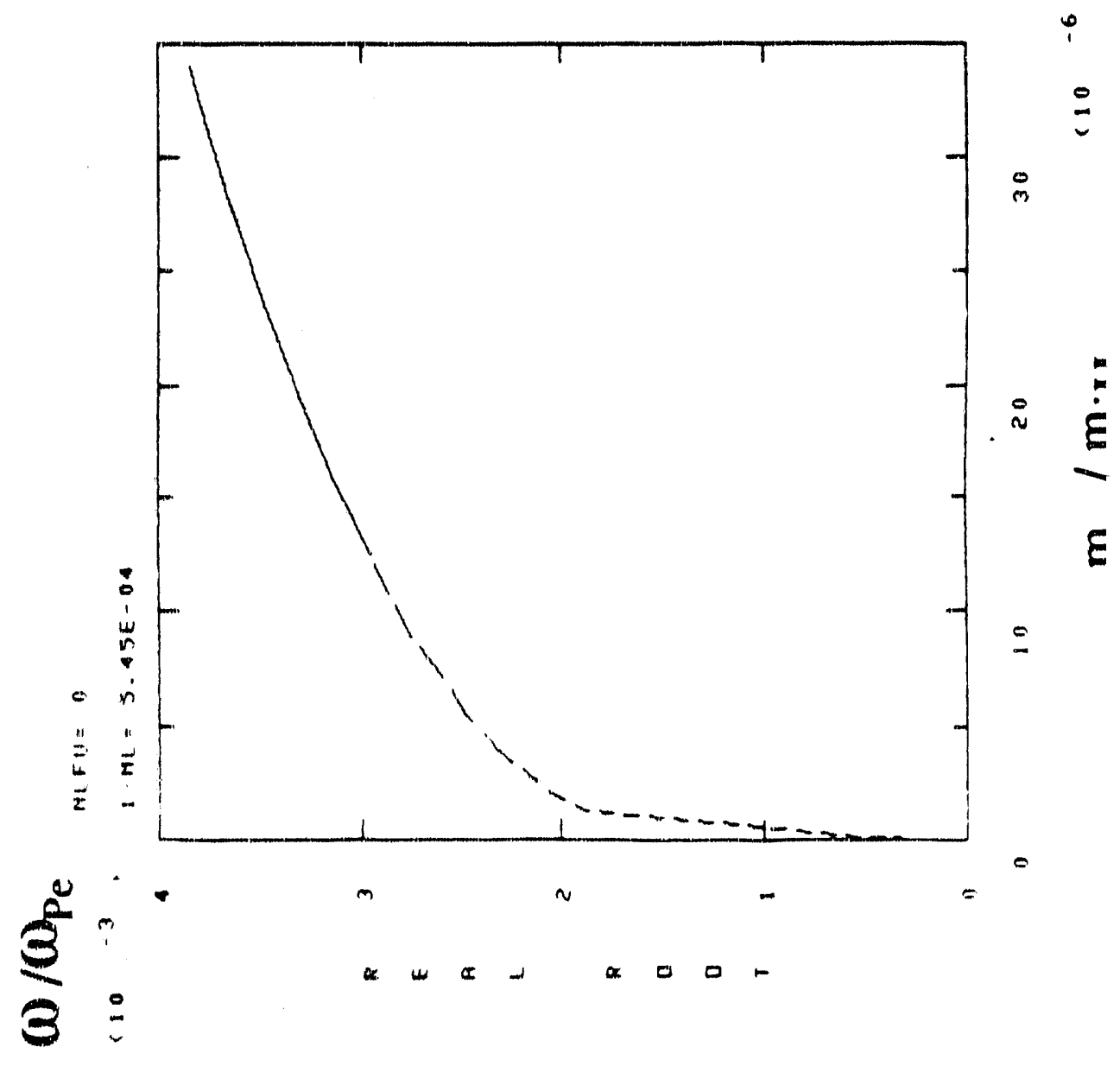




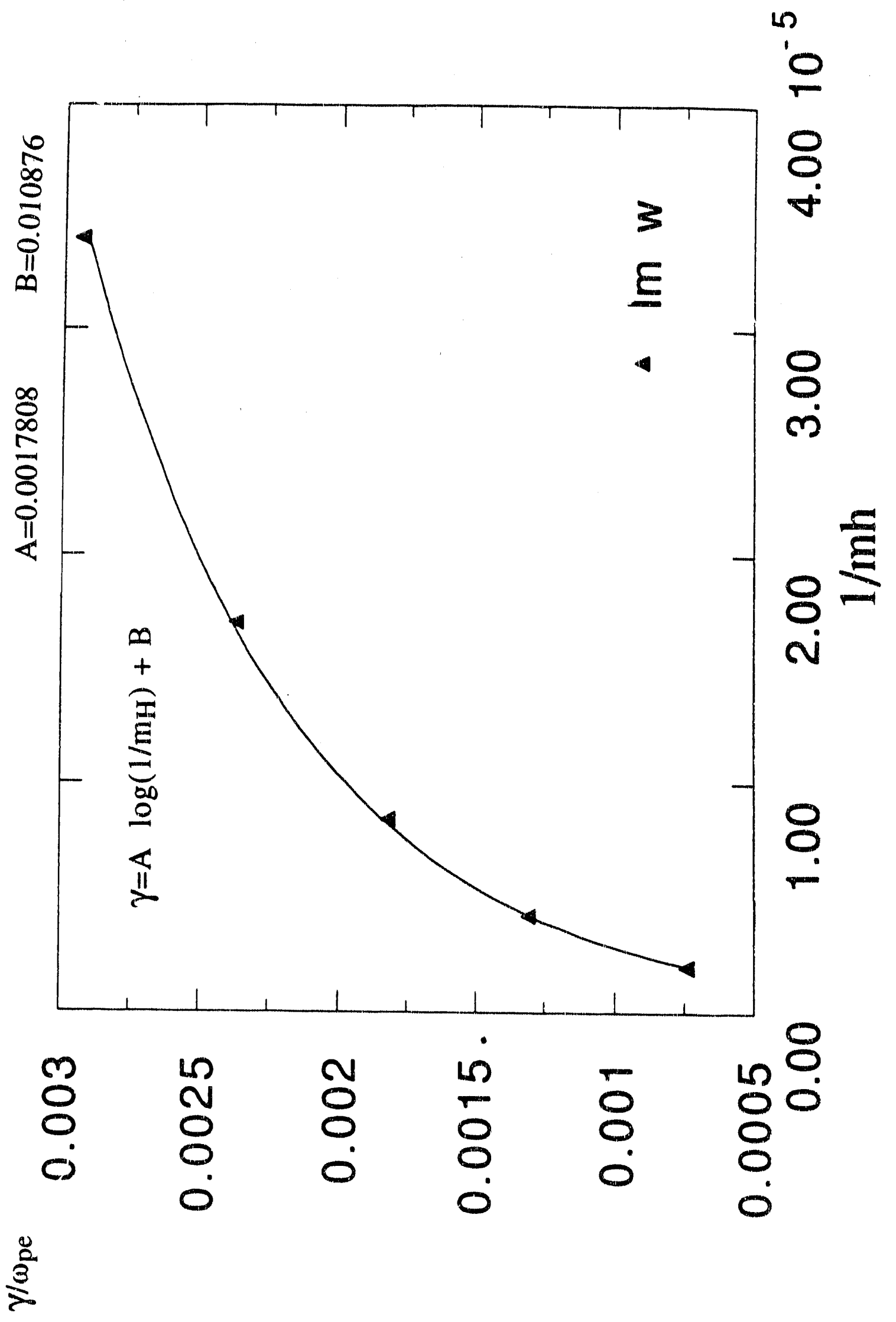

0
0
0 


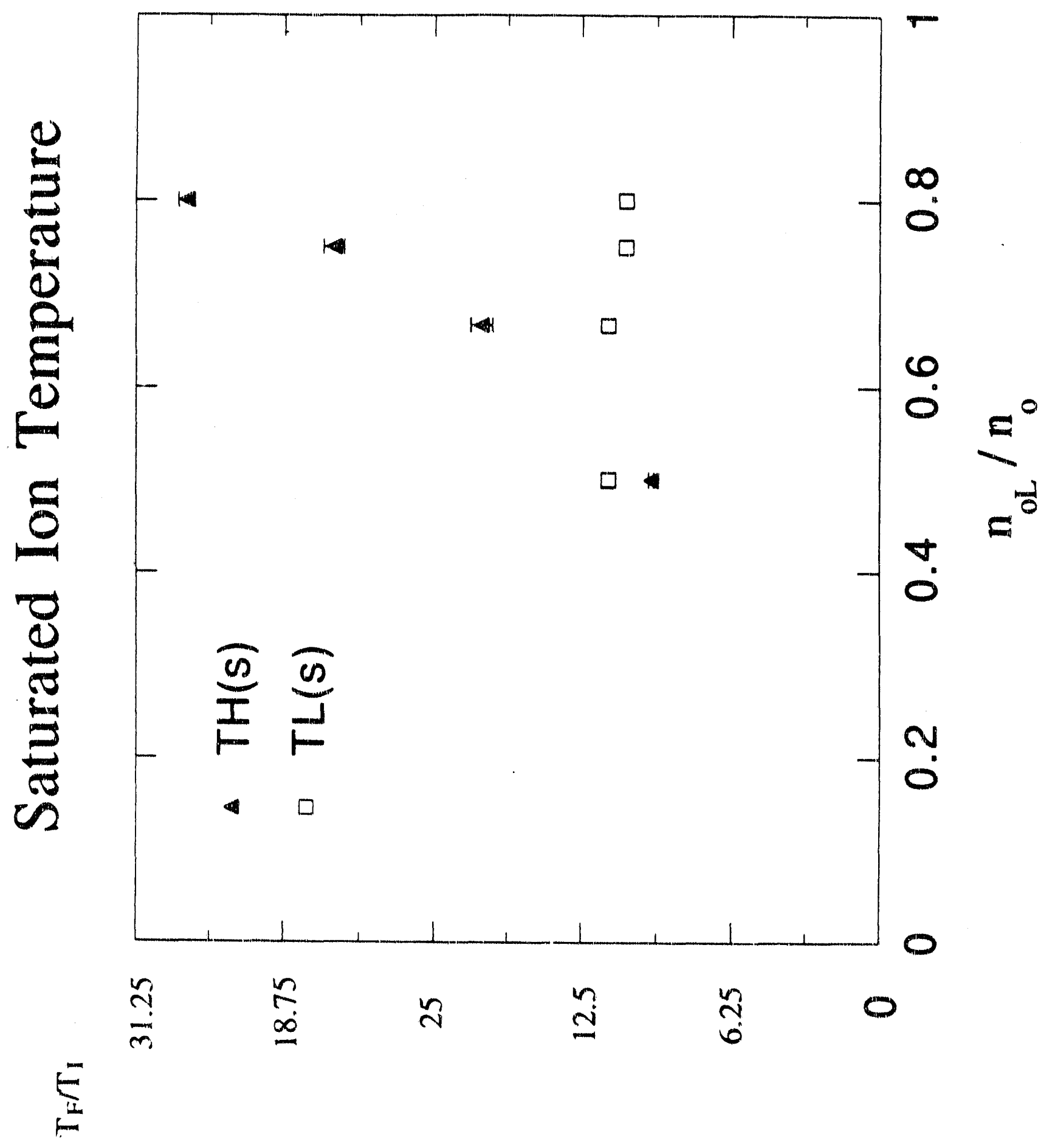




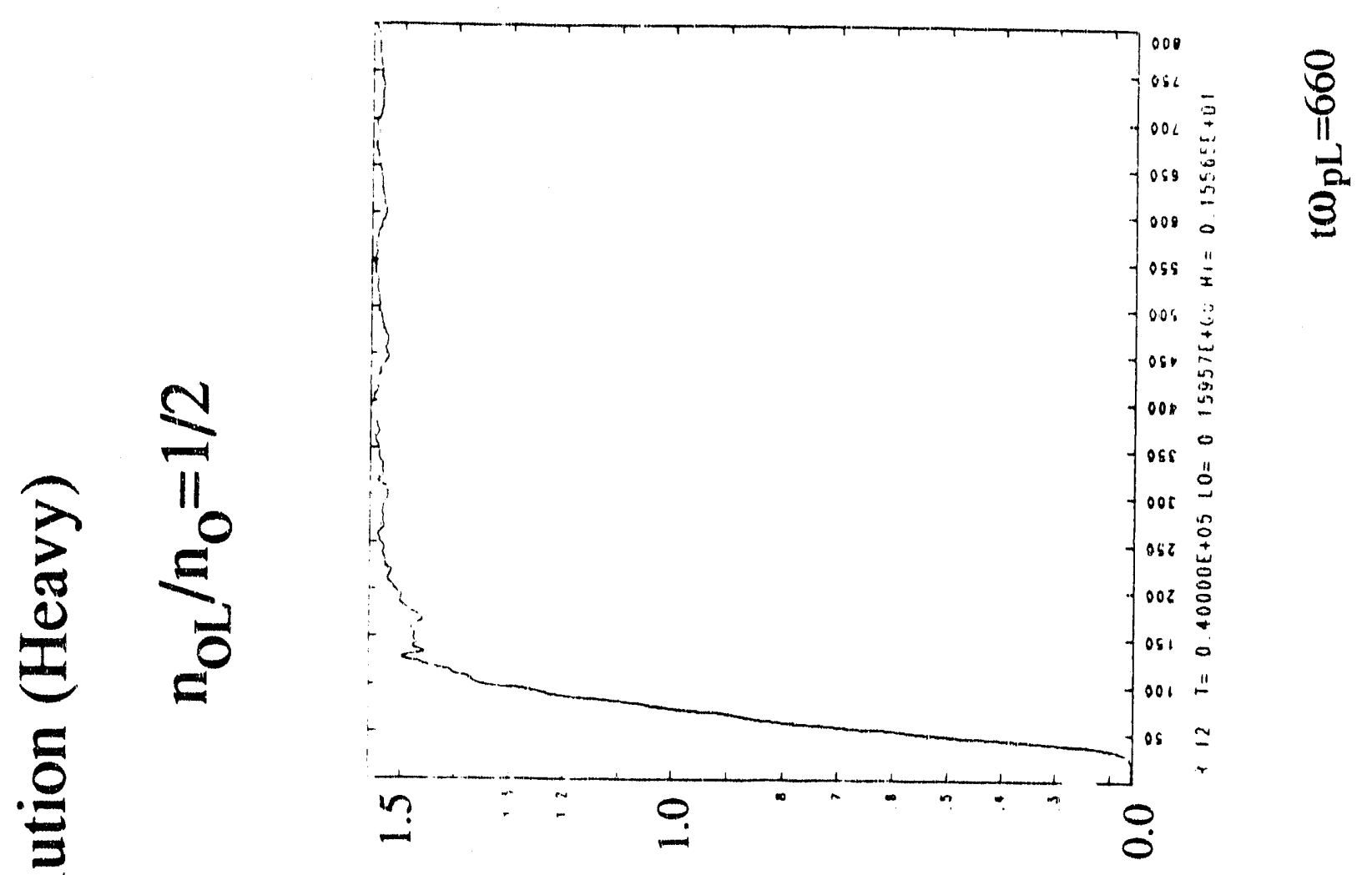

(r)

$\frac{0}{0}$
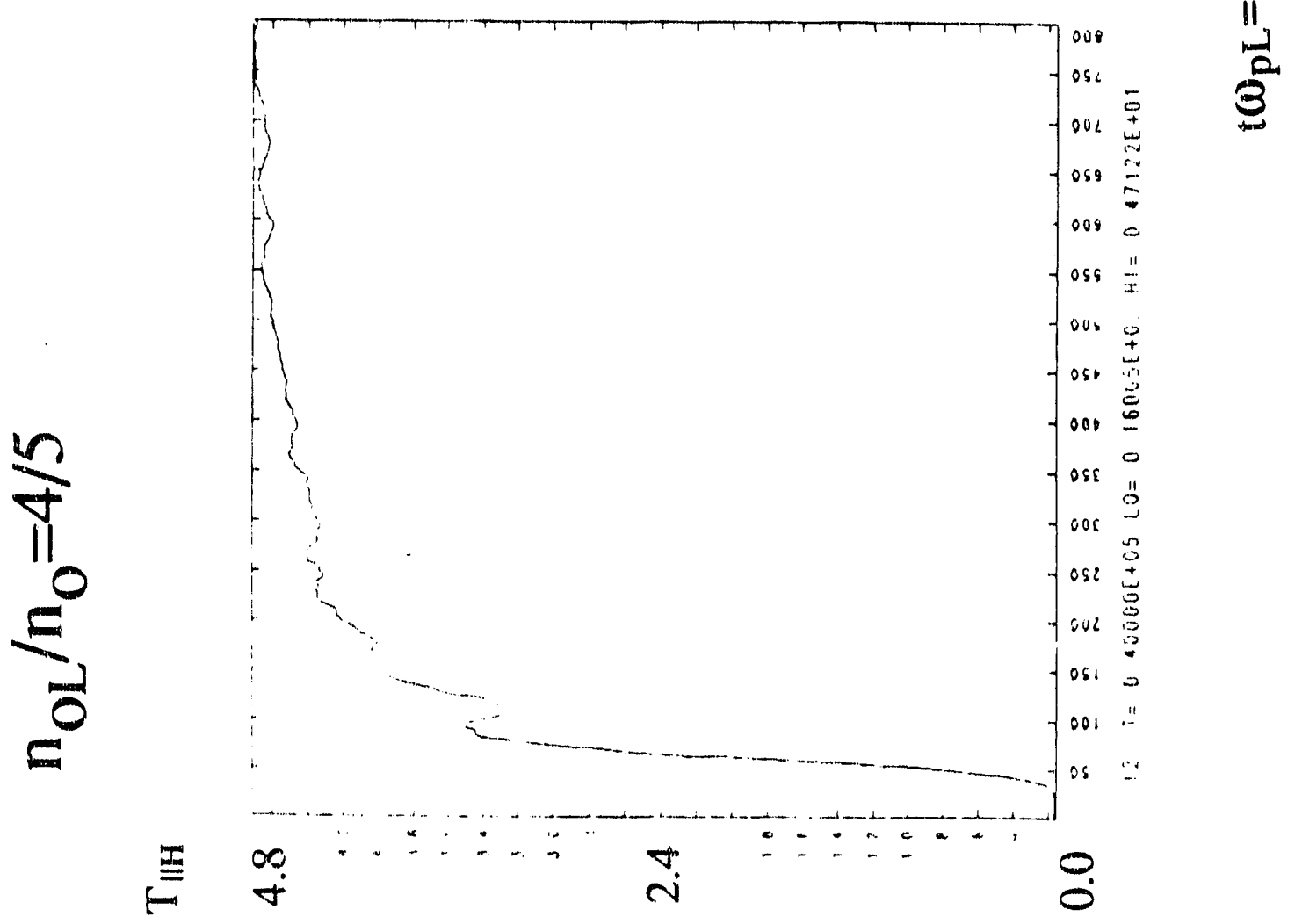


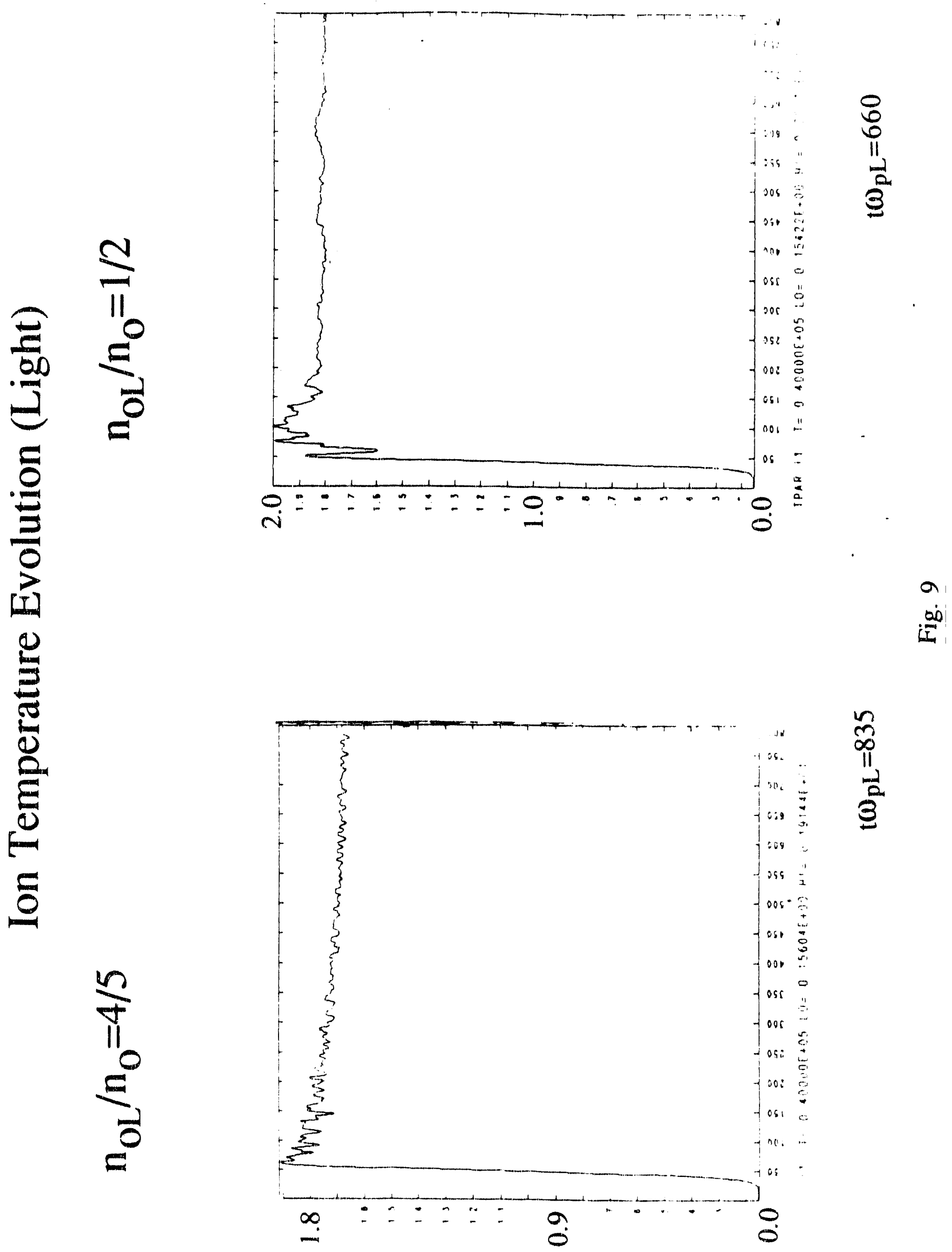



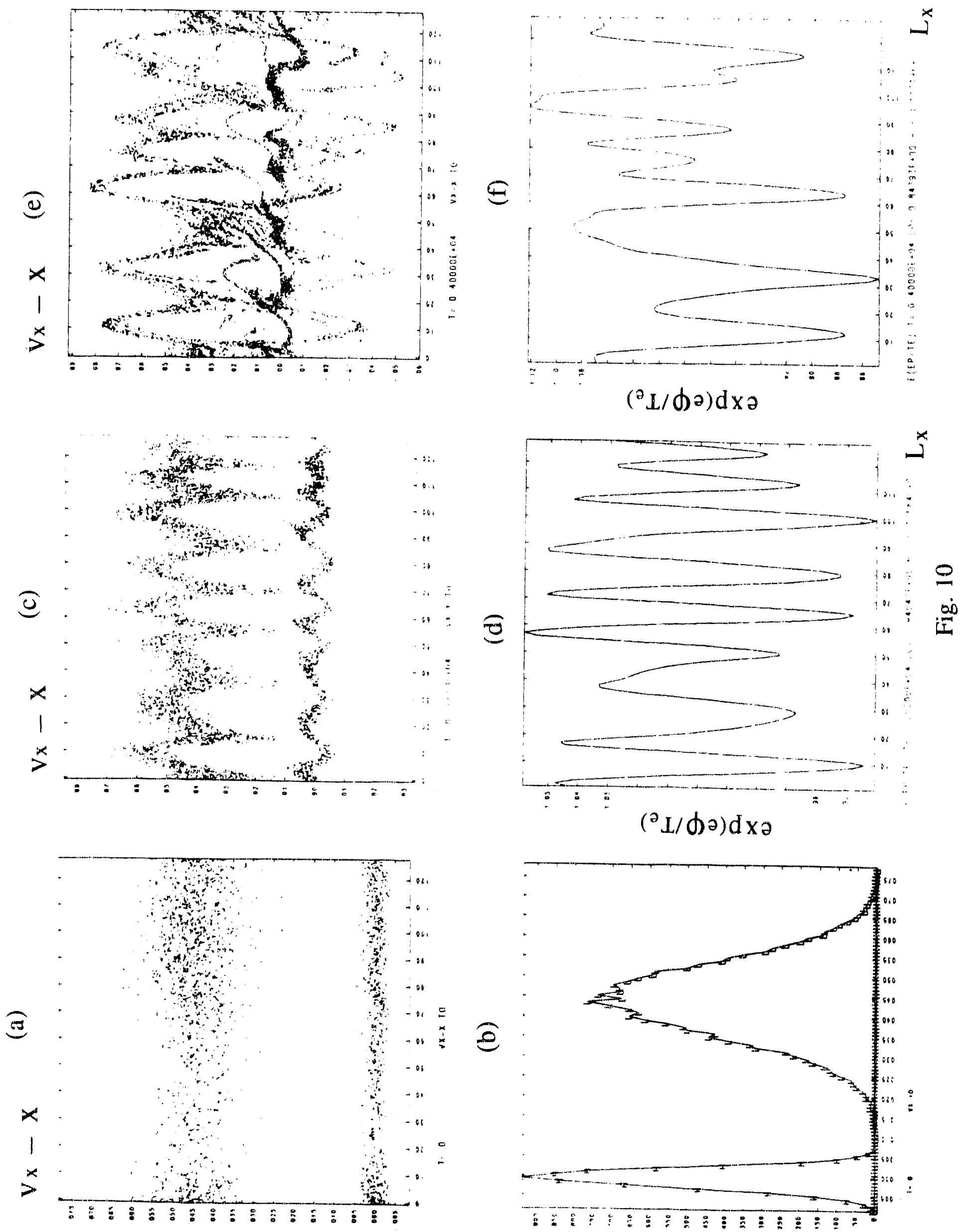
(g)

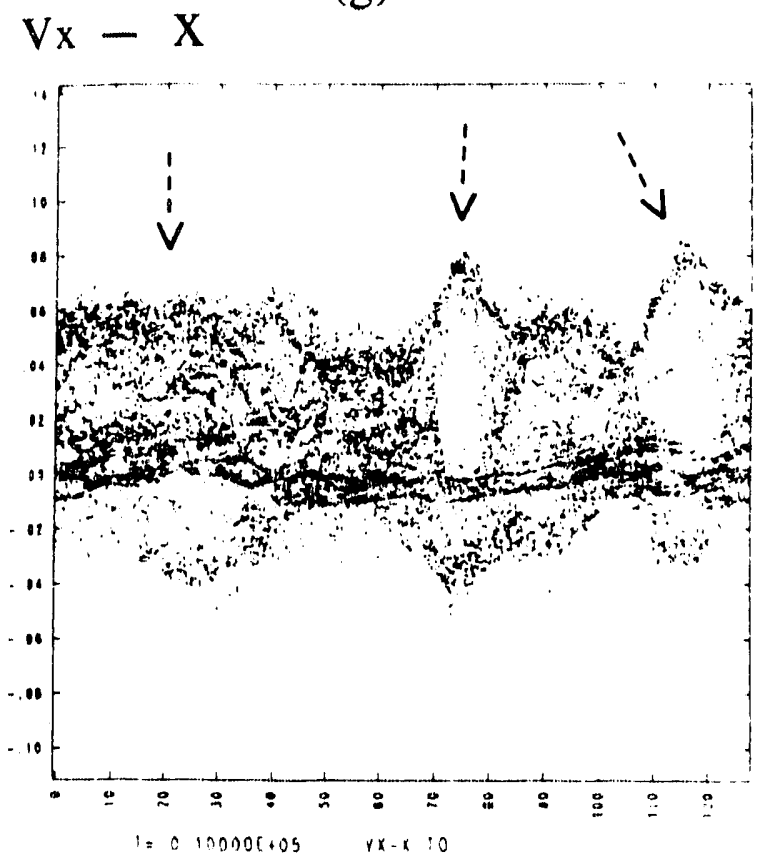

(h)

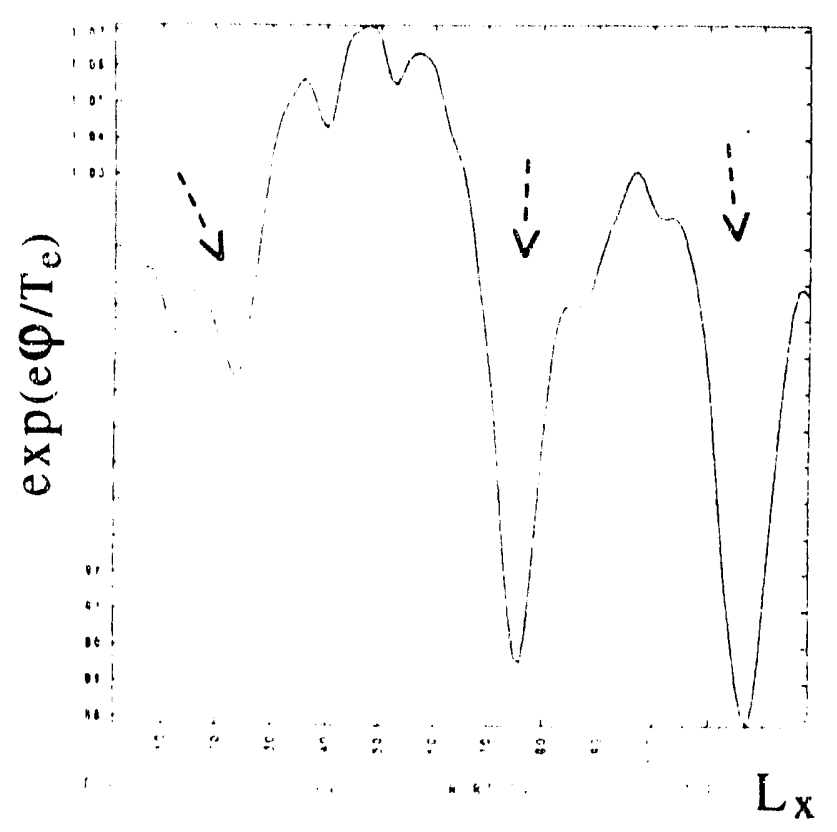

$V x-X$

(i)

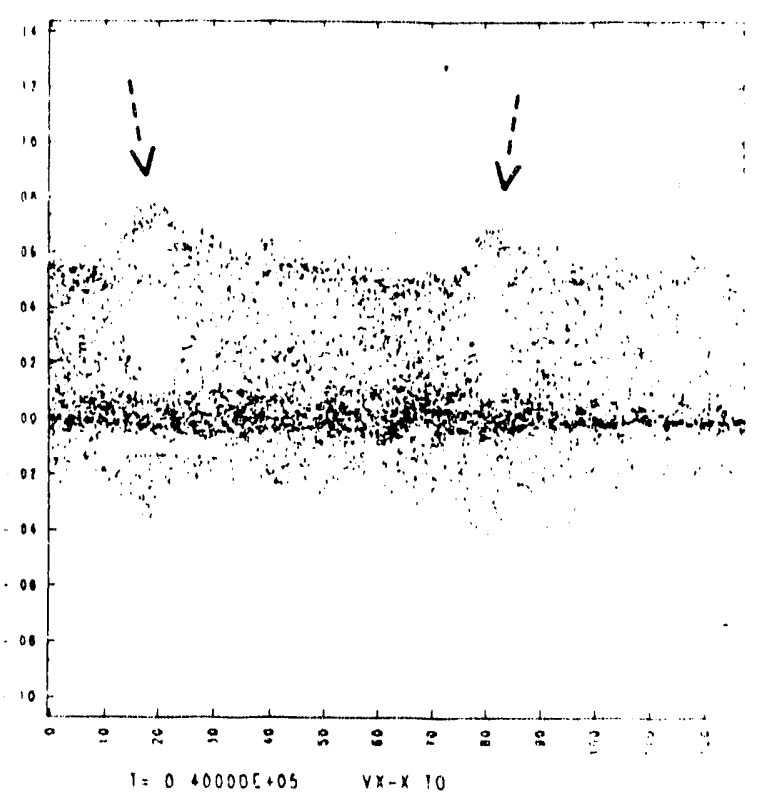

(j)

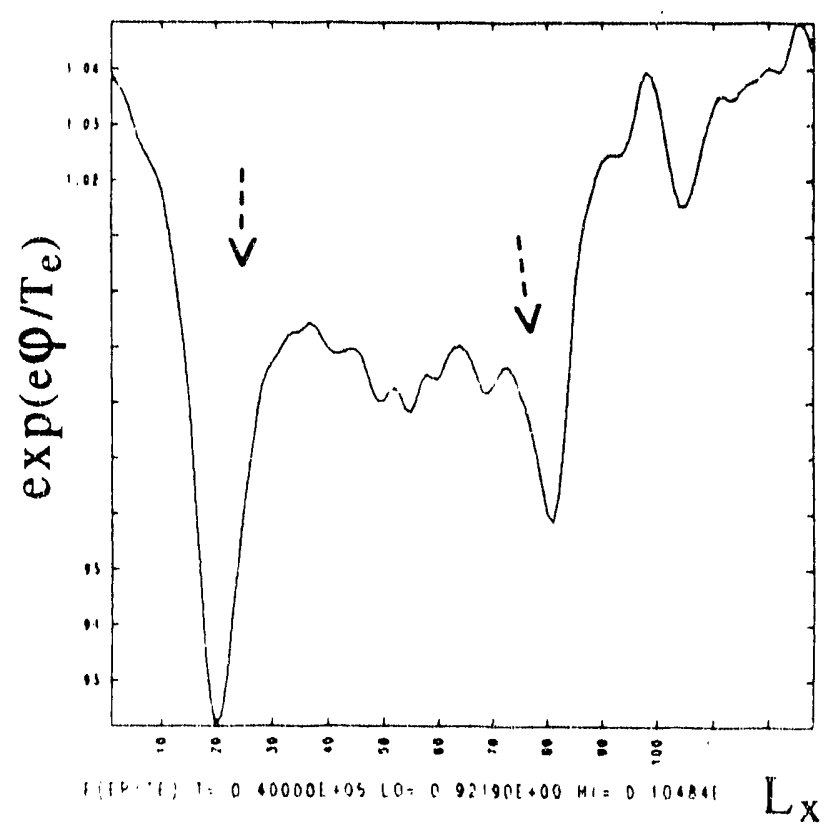

Fig. 11 

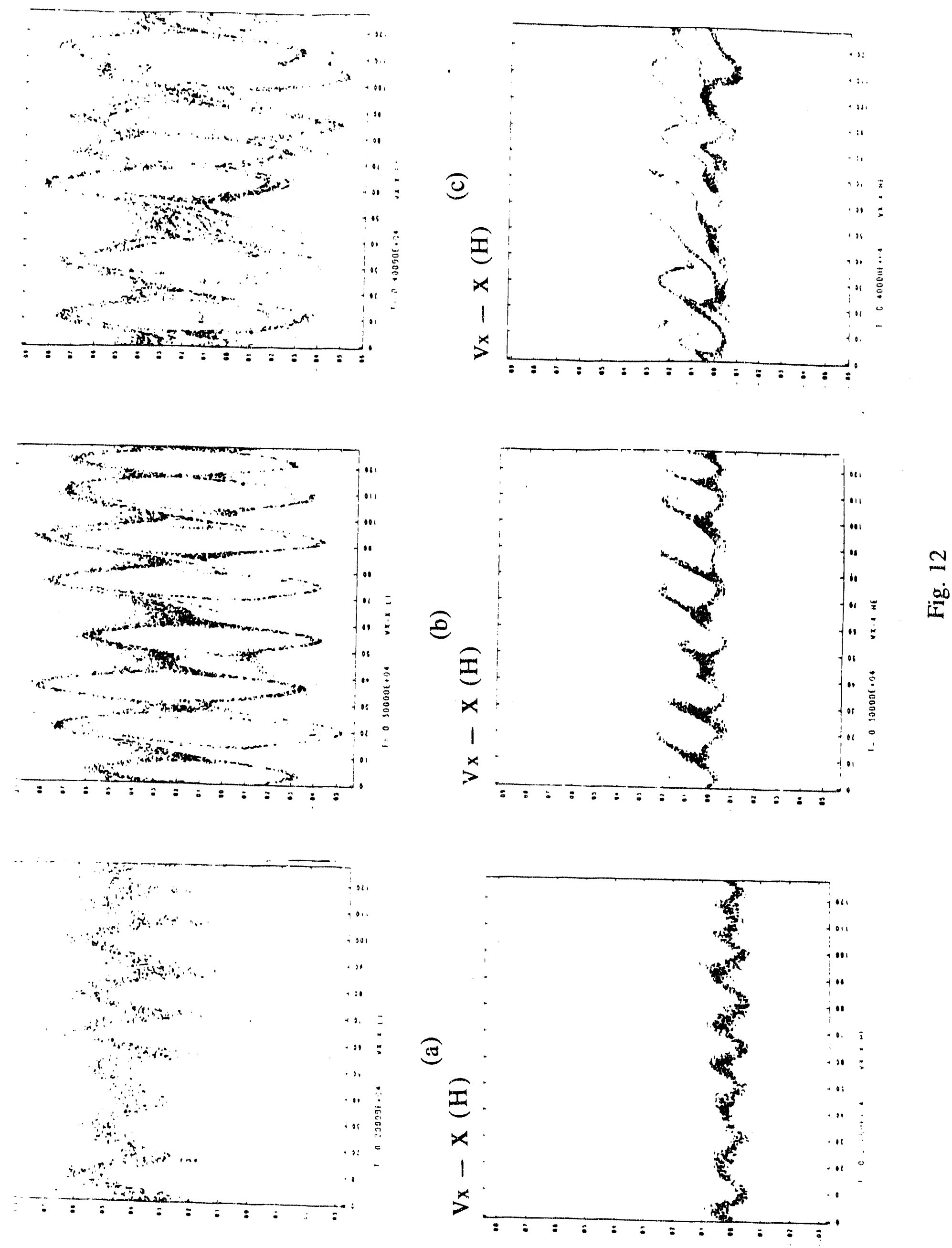

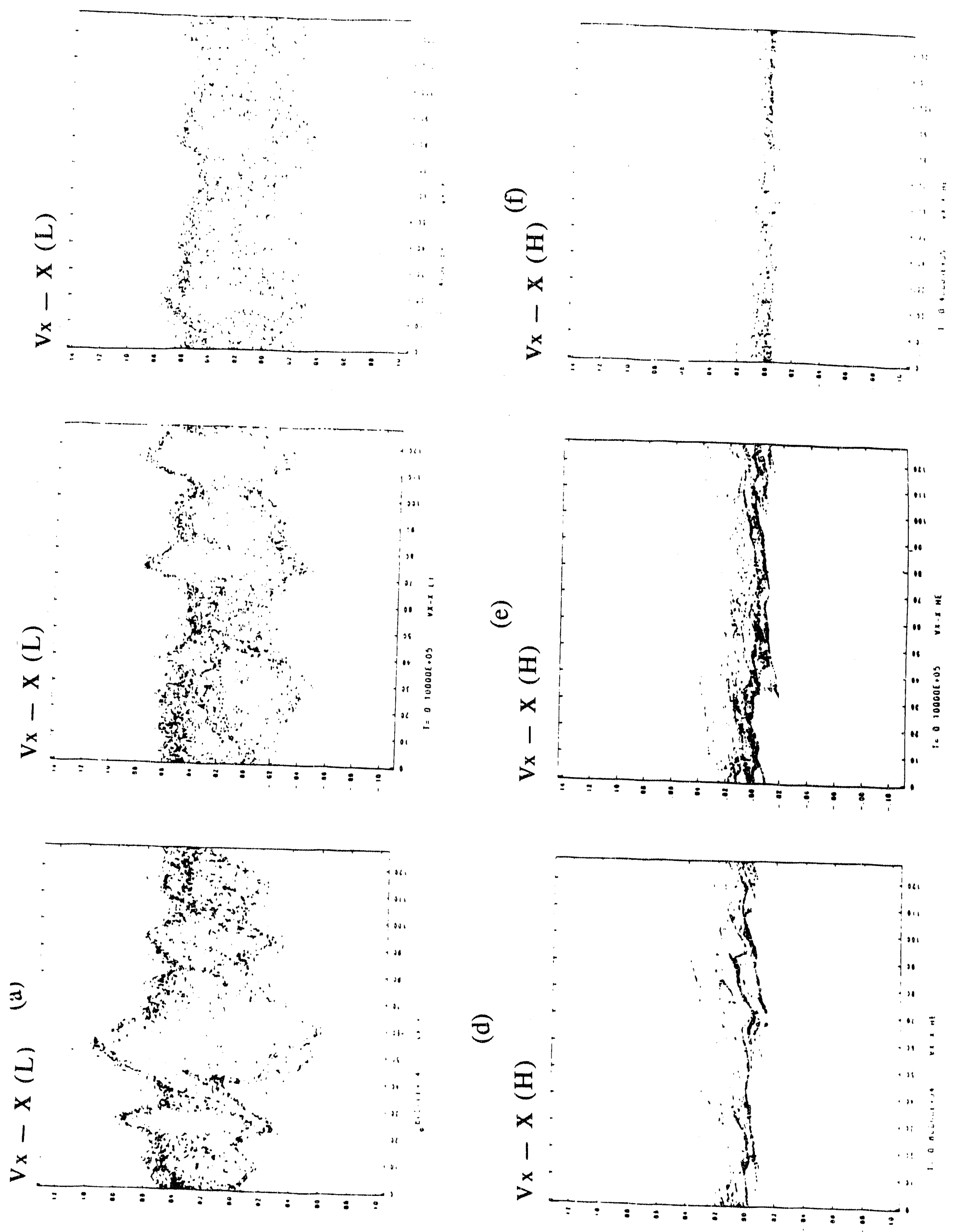

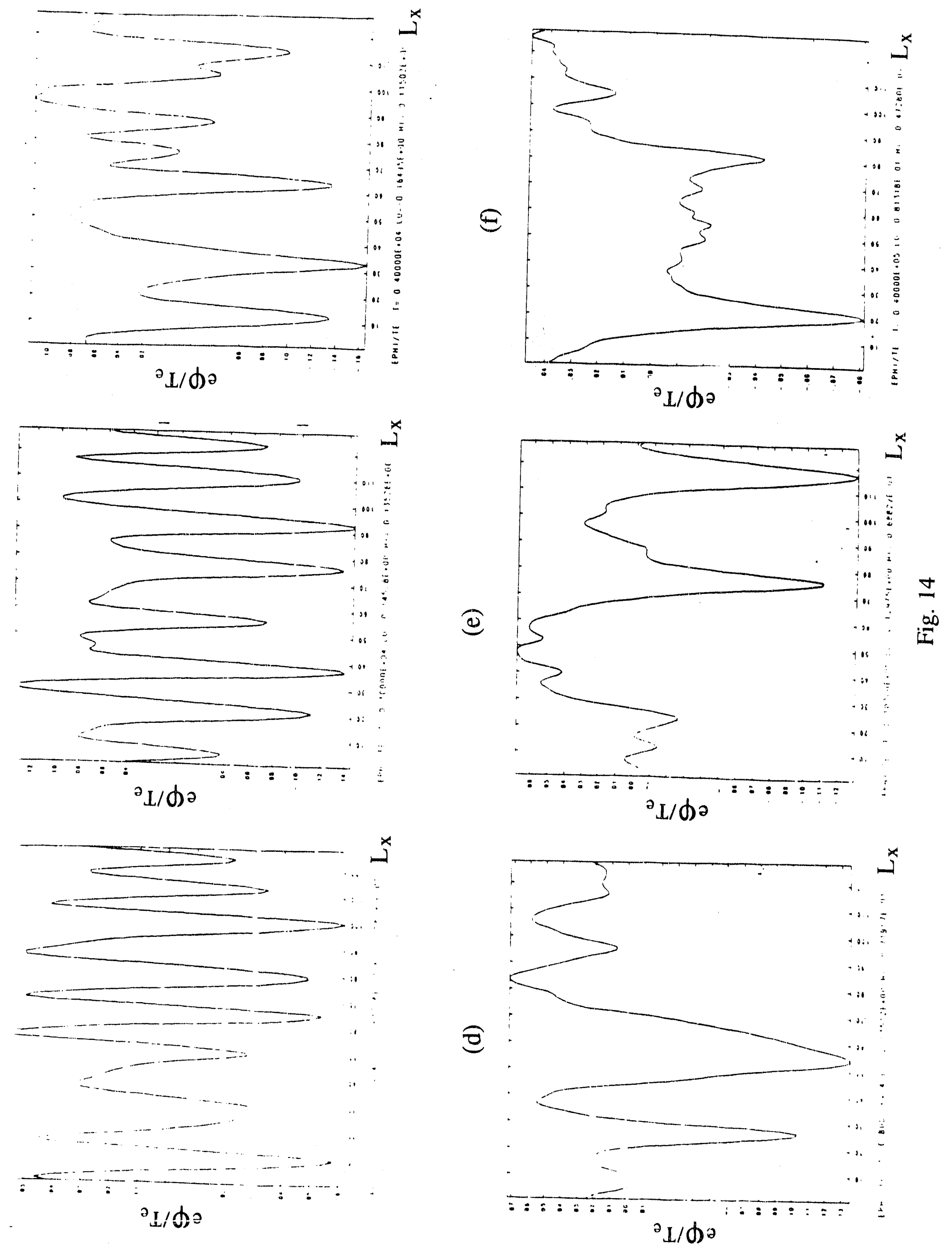

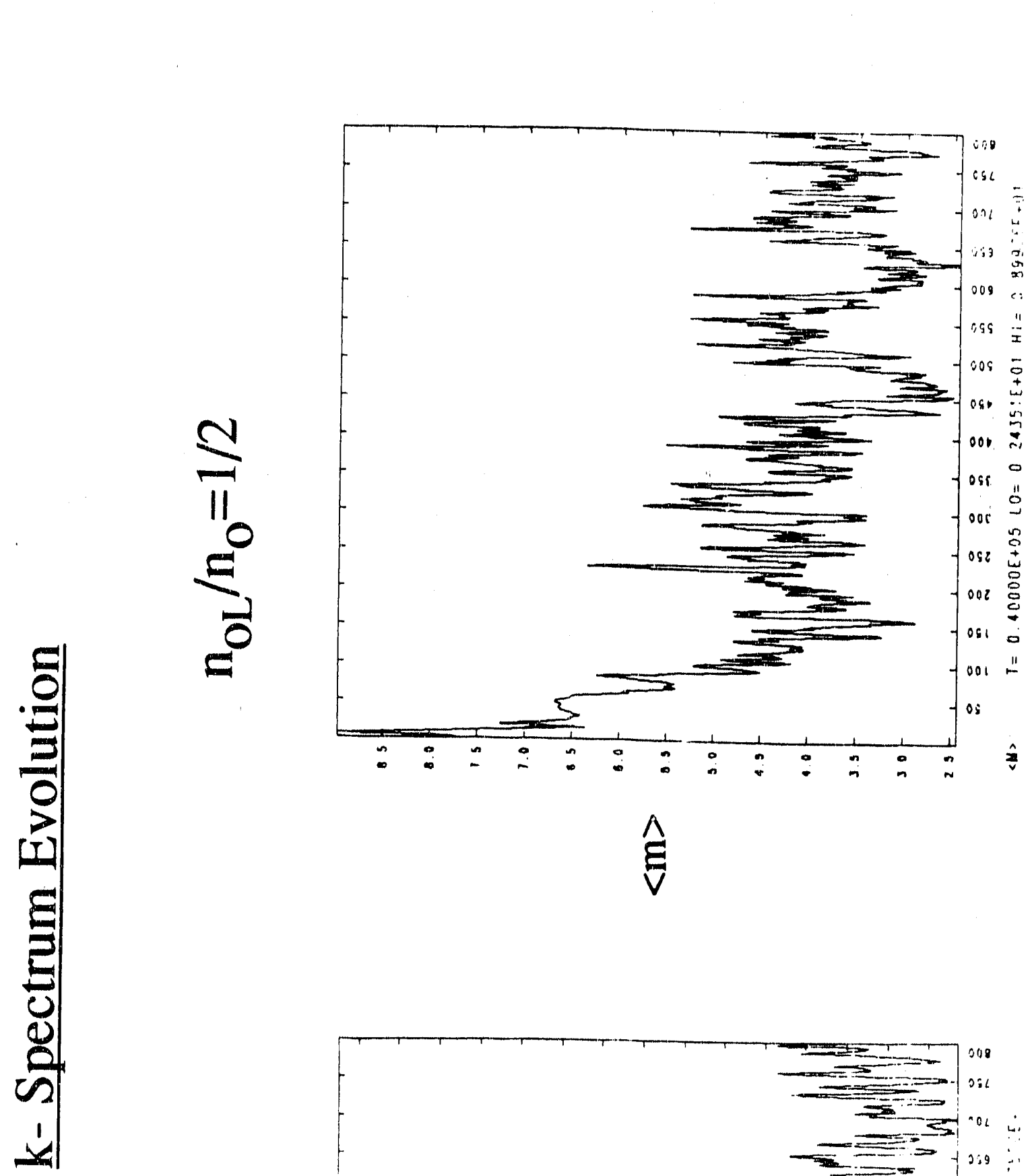

8

3

$\hat{V}$

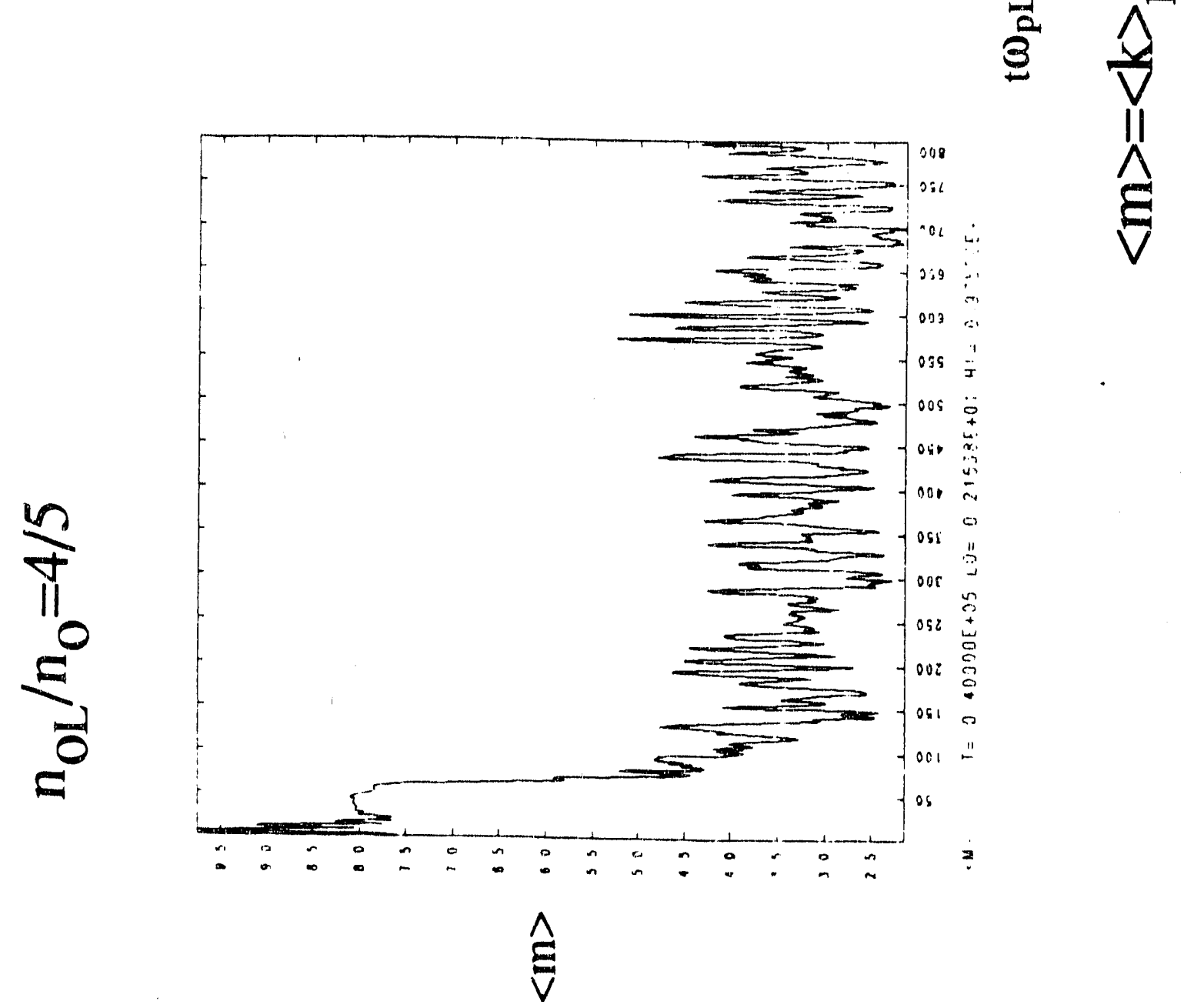

$\infty$ 


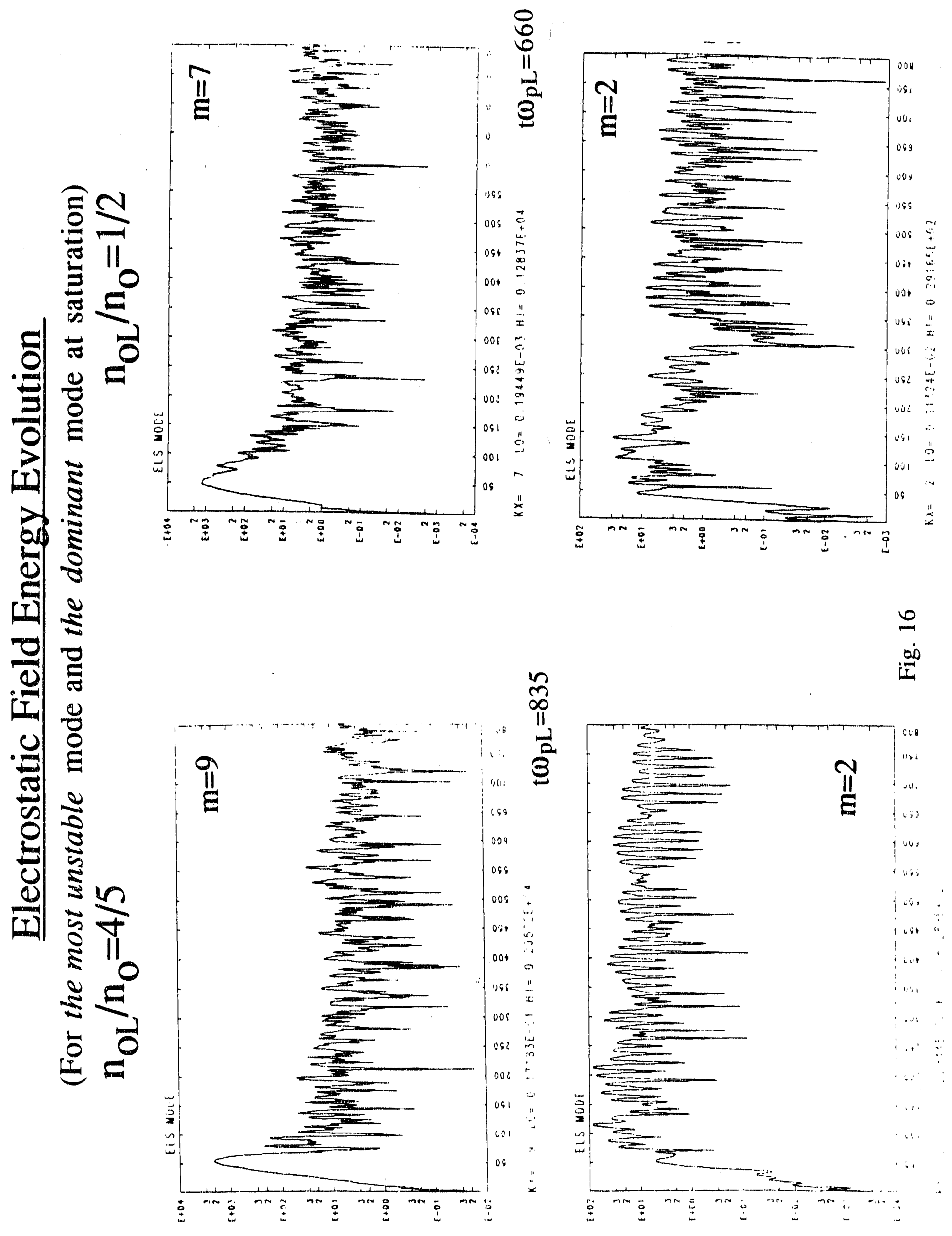



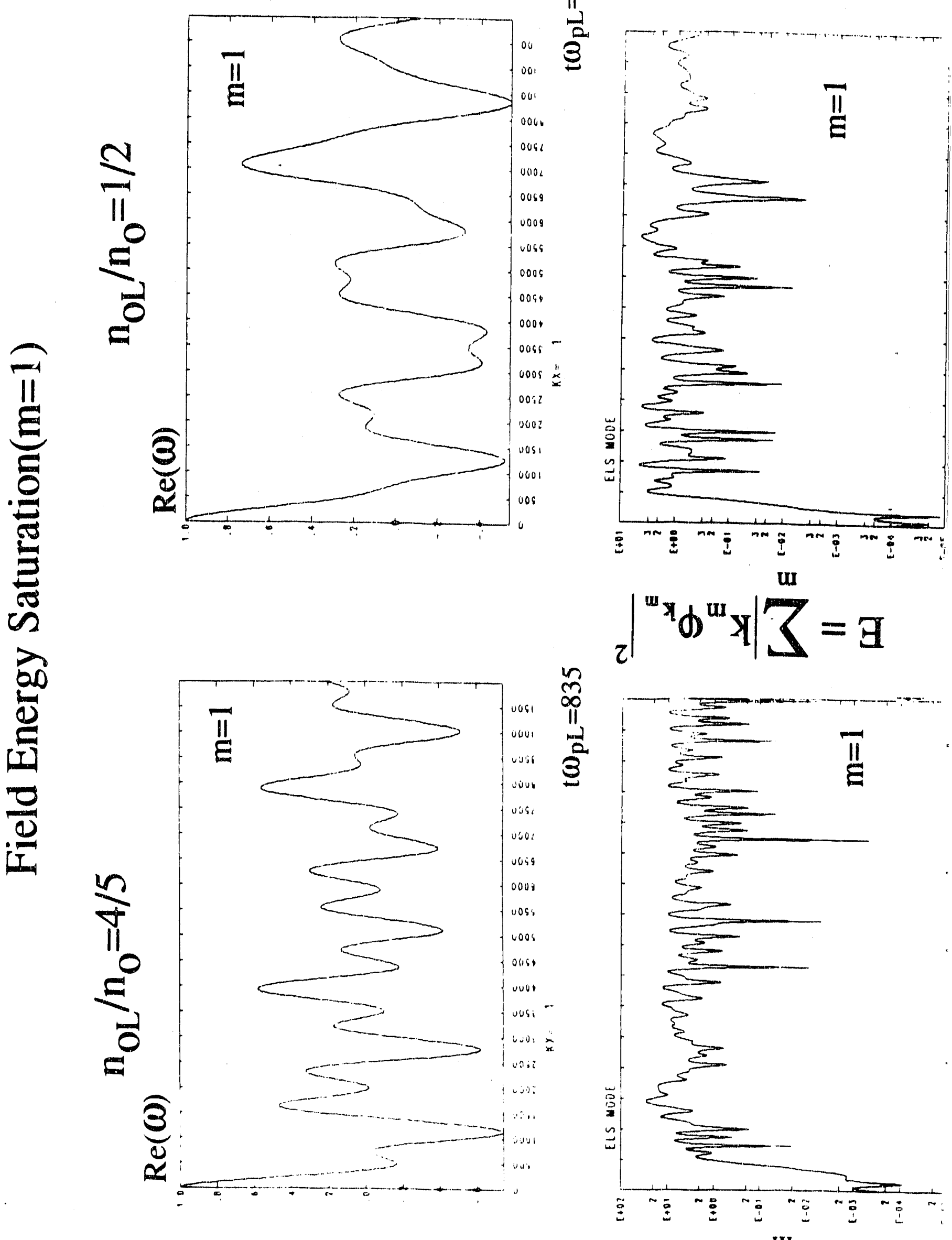

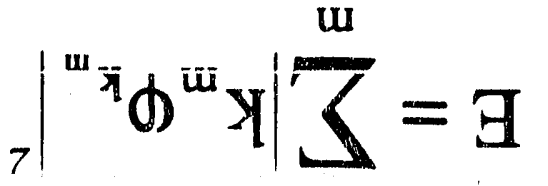




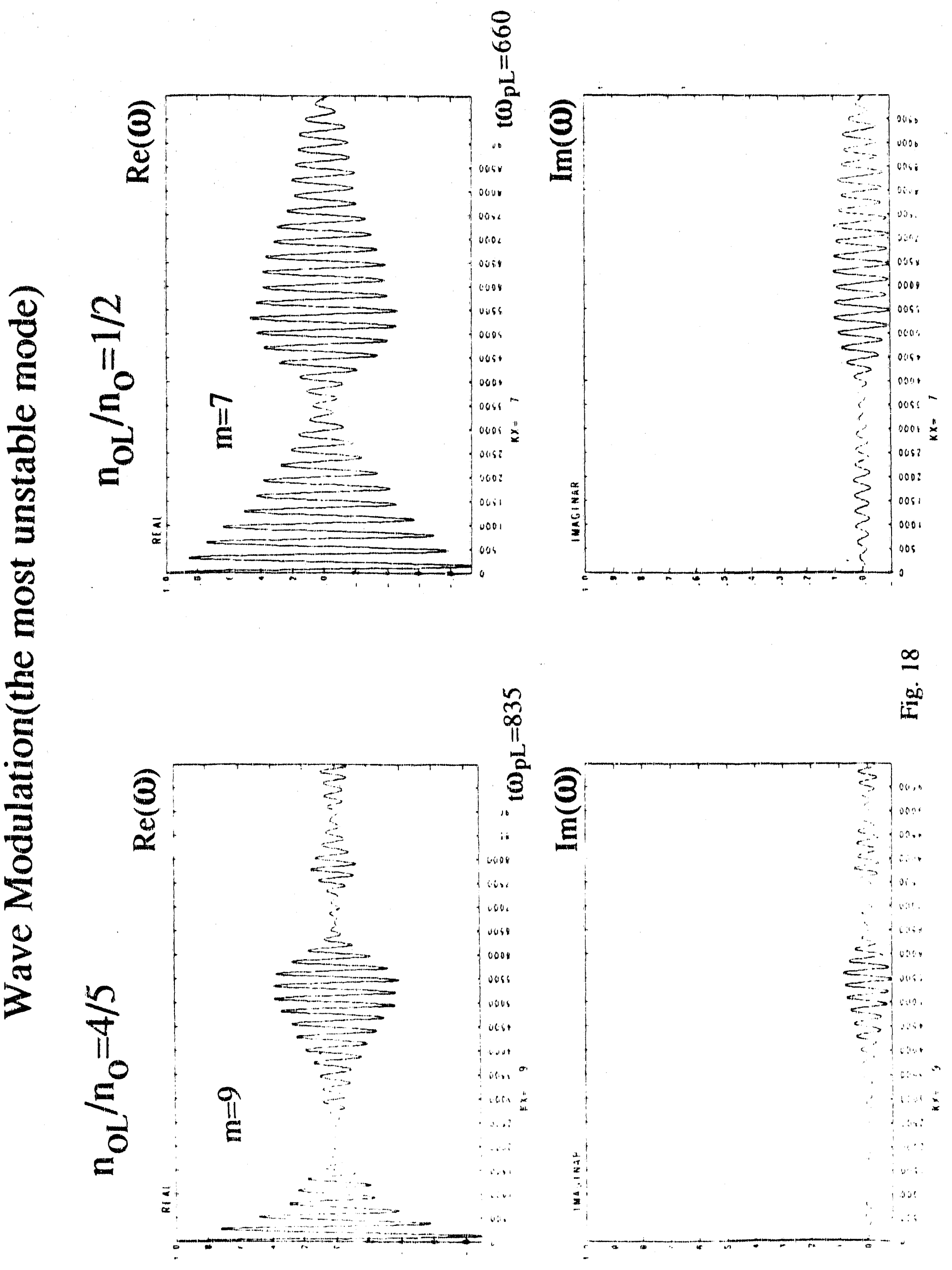




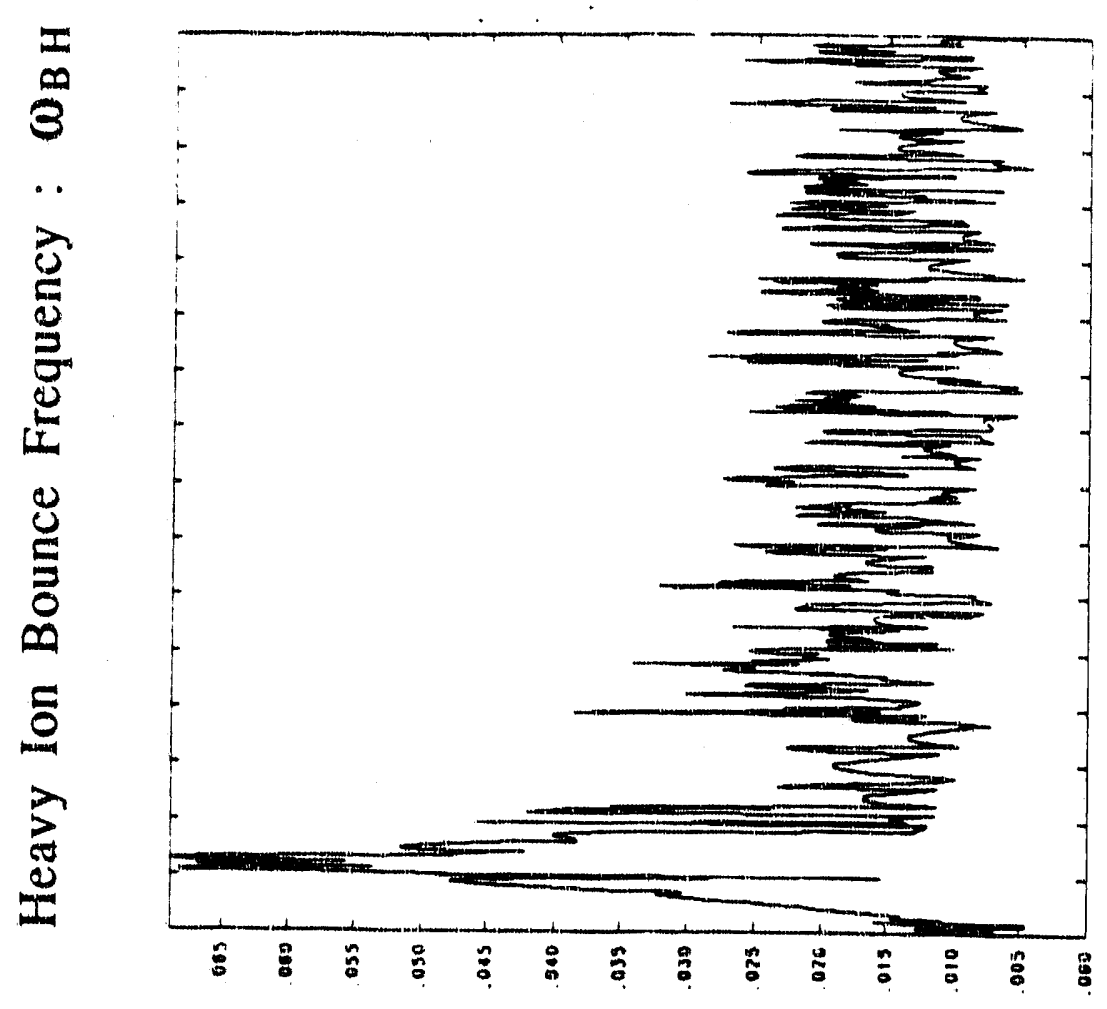

$\bigotimes_{\infty}^{\infty}$

$a$

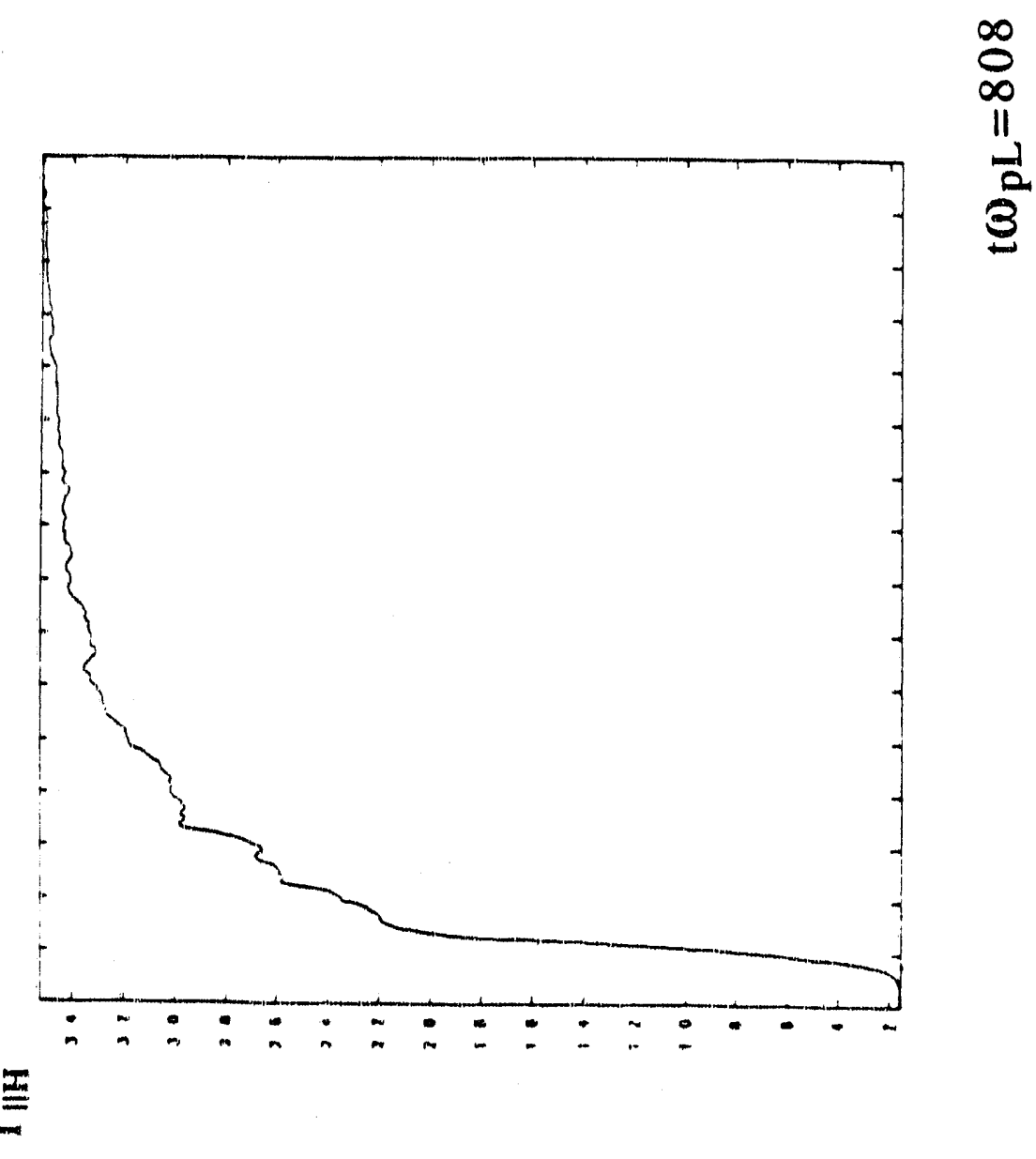




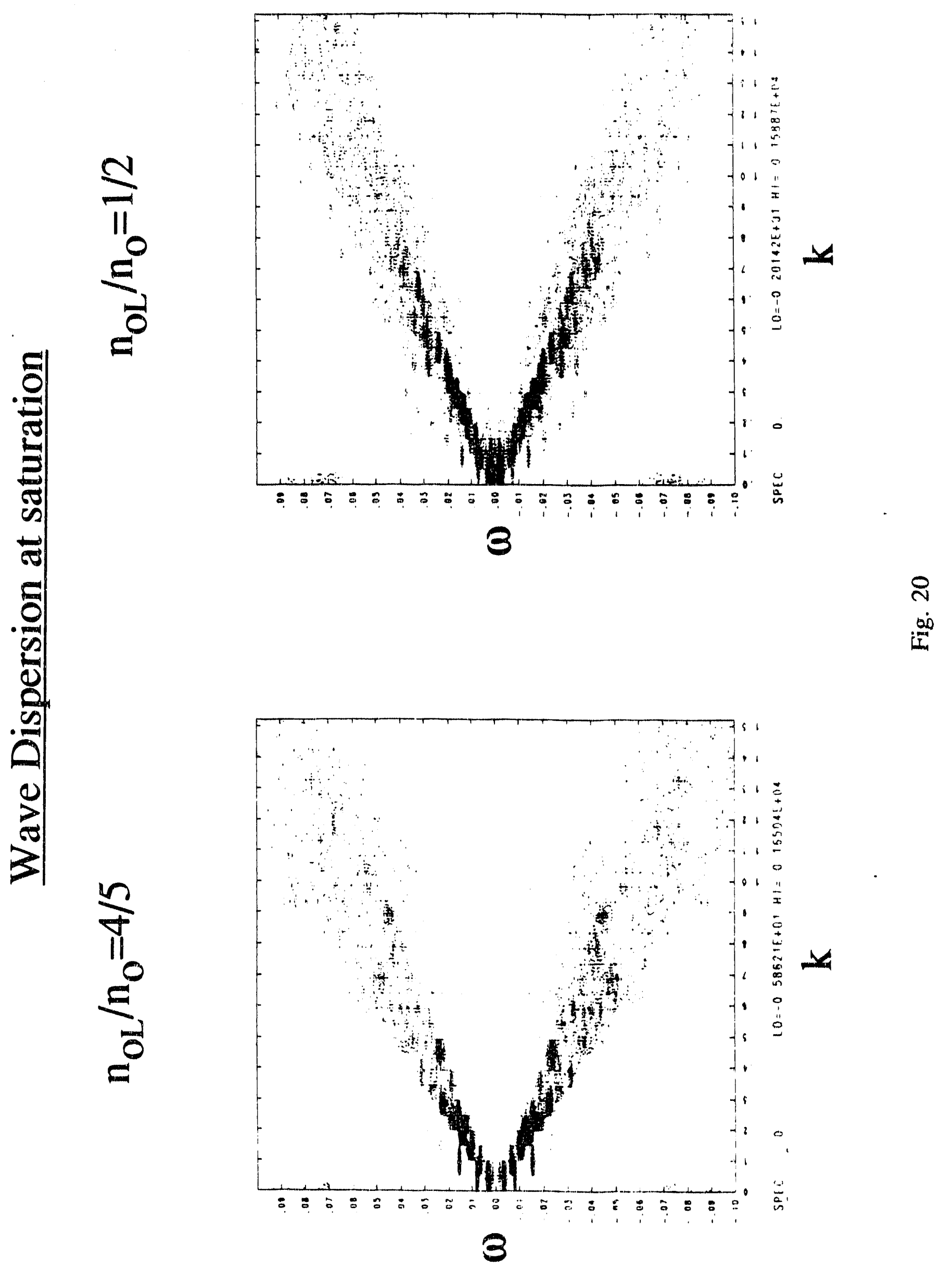




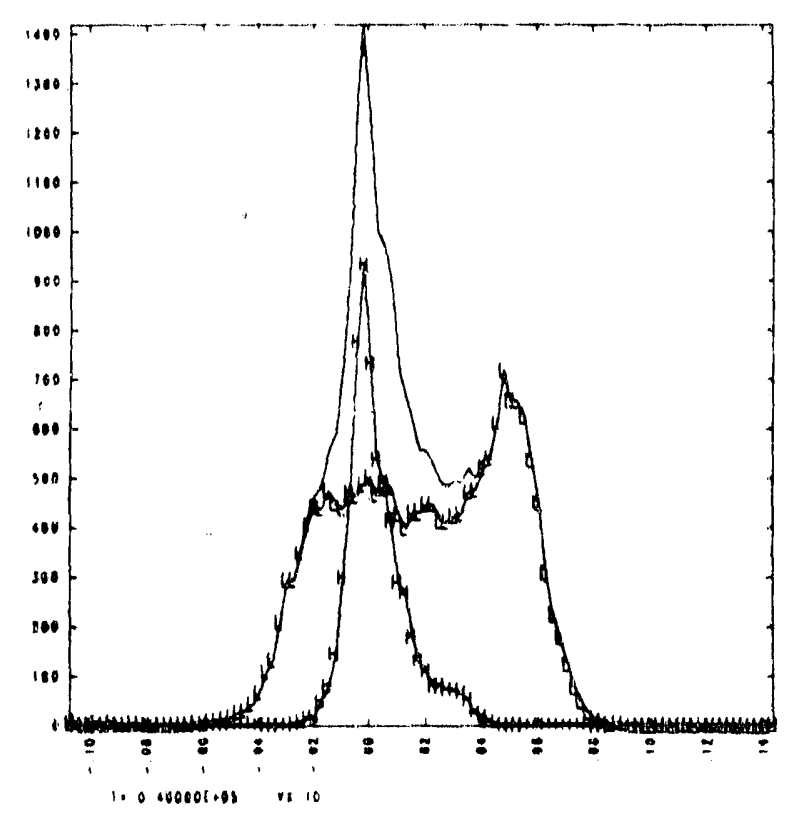

Fig 21. 

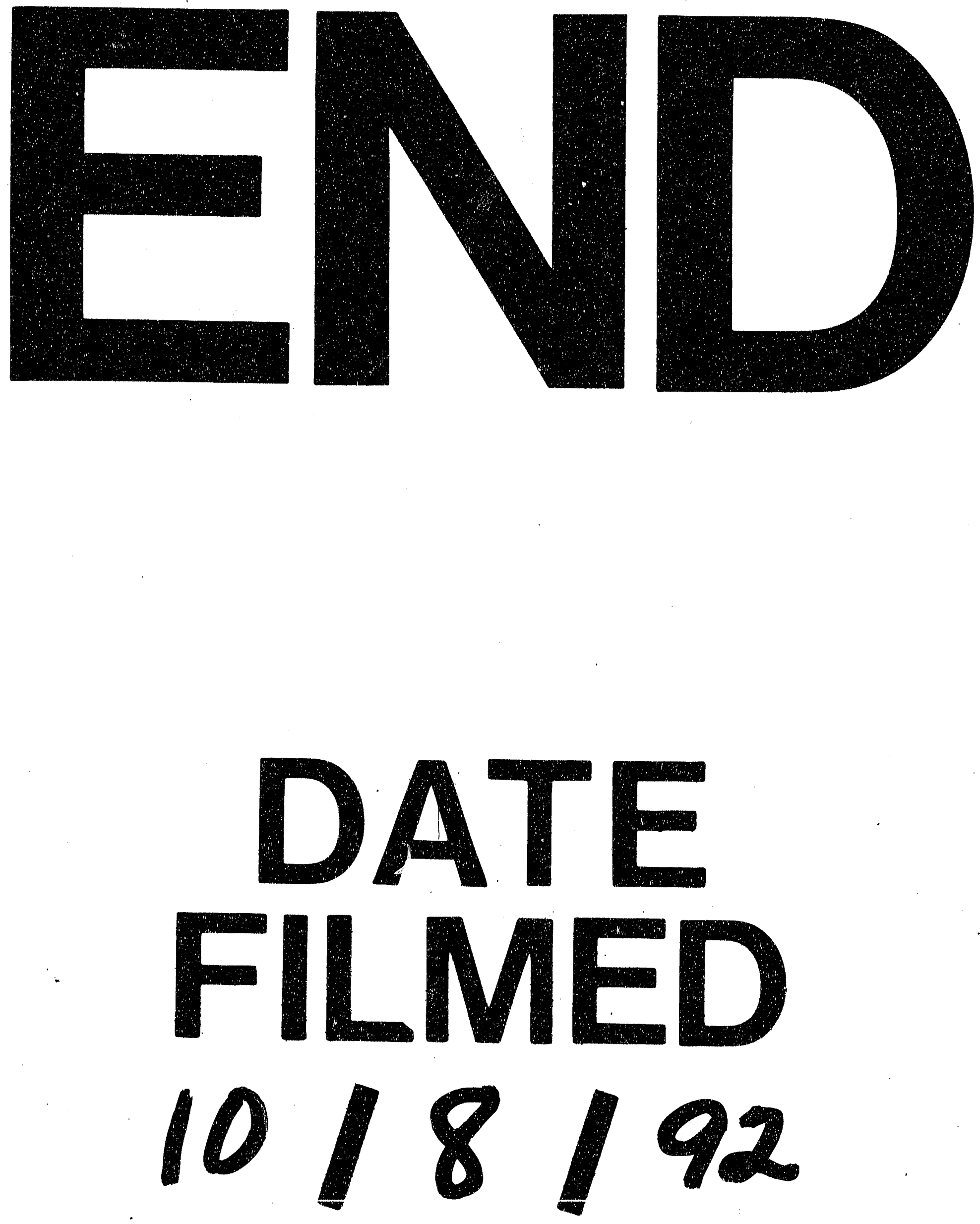
